Gerión. Revista de Historia Antigua

ISSN: 0213-0181

http://dx.doi.org/10.5209/geri.65984

\title{
Democrazia e impero ad Atene nella History of Greece di George Grote
}

\author{
Carlo Marcaccini ${ }^{1}$
}

Recibido: 27 de marzo de 2019 / Aceptado: 23 de abril de 2019

Riassunto. Nella History of Greece George Grote celebra la democrazia ateniese e la considera un regime politico esemplare. Grote disprezza l'oligarchia e ritiene che la sovranità popolare ateniese abbia posto fine alle lotte di fazione. In particolare, la riforma periclea dei tribunali garantì il rispetto della legge e impedì ai più forti e ai più ricchi di prendere il potere. Tuttavia, $\mathrm{i}$ tribunali popolari furono anche un efficace sistema per controllare e governare i sudditi dell'impero marittimo. Grote paragona il dominio ateniese all'impero britannico: entrambi sono governments of dependencies, in cui gli stati sudditi gestiscono liberamente i loro affari interni, ma dipendono dallo stato dominante nella politica estera. Le controversie fra Atene e i suoi alleati erano discusse nei tribunali ateniesi nei quali, secondo Grote, i greci del tempo trovarono giustizia e clemenza, e furono così ricompensati per la perdita della loro autonomia.

Parole chiave: George Grote; democrazia antica; tribunali popolari; impero marittimo ateniese.

\section{[en] Democracy and Empire in Athens in George Grote's History of Greece}

Abstract. In his History of Greece, George Grote celebrates the Athenian democracy and considers it an exemplary political regime. Grote despises oligarchy and believes that Athenian popular sovereignty has put an end to factional struggles. In particular, the reform of the courts ensured respect for the law and prevented the strongest and richest from taking power. However, the popular dikasteries were also an effective system for controlling and governing the subjects of the maritime empire. Grote compares the Athenian rule with the British Empire: both are governments of dependencies, in which the subdued states freely manage their internal affairs, but depend on the dominant state in foreign policy. The disputes between Athens and its allies were discussed in the Athenian courts where, according to Grote, the Greeks found fairness and tolerance, and were thus rewarded for the loss of their autonomy.

Keywords: George Grote; Ancient Democracy; Popular Dikasteries; Athenian Empire.

Sumario. 1. Introduzione. 2. Una storia personale e universale. 3. Schömann vs Grote. 4. I tribunali popolari. 5. Government of dependencies. 6. Conclusione. 7. Bibliografia.

Cómo citar: Marcaccini, C. (2019): Democrazia e impero ad Atene nella History of Greece di George Grote, en Gerión 37/2, 489-514.

1 Scuola militare aeronautica "Giulio Douhet" (Firenze).

E-mail: carlo.marcaccini@gmail.com 


\section{Introduzione ${ }^{2}$}

Frank Turner, in un saggio fondamentale sulla tradizione classica in Inghilterra, scrive che per gli inglesi di età vittoriana "the Greeks had been like the Victorians and that the historical situations of the two civilizations were essentially similar". ${ }^{3}$ I vittoriani si entusiasmavano per le grandi imprese dei greci e si identificavano negli eroi di Maratona, di Salamina, più che nei loro antenati ad Agincourt, a Trafalgar, a Waterloo. ${ }^{4}$ In questa moderna mitopoiesi il modello classico era vivo e attivo, soprattutto in politica. Già nel 1831, George Cornewall Lewis, sul quale tornerò più avanti, scrisse a Karl Otfried Müller:

In England politics engross so much of the attention of all that few of our histories are not corrupted by temporary allusions. Mitford's History of Greece was written, at the time of the French Revolution, in order to shew the evils of democracy, and the author goes so far in his love of absolute kings and hatred of republics, that he is inclined to set the Macedonians above the Athenians! $!^{5}$

Dopo Mitford anche altri studiosi intrecciarono politica e storia, trovando nel passato una fonte di ispirazione e di propaganda, uno specchio in cui guardarsi e rimirarsi, anche con un certo compiacimento. Questo riflesso è particolarmente smagliante nella History of Greece di Grote, che per originalità e chiarezza divenne un punto di riferimento per gli studi classici sia in Inghilterra sia in Europa, in particolare in Germania. ${ }^{6}$ Ciò che colpì e scandalizzò i contemporanei era lo smaccato atenocentrismo di Grote e la sua esaltazione della democrazia antica, un aspetto che attrae la nostra attenzione anche oggi. ${ }^{7}$ Tuttavia nella History la democrazia ateniese non era solo un modello di partecipazione politica e di moralità pubblica, ma anche uno strumento di potere, attraverso il quale gli ateniesi, facendo prevalere la giurisdizione dei loro tribunali in tutto l'impero, gestivano i rapporti con gli stati sottomessi. Era soprattutto questa l'Atene in cui l'Inghilterra vittoriana amava specchiarsi, in cui vedeva il suo riflesso più autentico. ${ }^{8}$

\section{Una storia personale e universale}

George Grote non fu un accademico, ma un banchiere, socio della Prescott, Grote \& Co., fondata dal padre, al quale subentrò nel $1828 .{ }^{9}$ Decise di ritirarsi dall'attività nel 1843, quando già aveva iniziato la stesura della History, in cantiere da molti anni. ${ }^{10}$

\footnotetext{
Desidero ringraziare Mirko Canevaro per i suoi preziosi suggerimenti, di cui darò conto nelle note. Turner 1981, 11. Sulla ricezione della tradizione classica in Inghilterra in età vittoriana: Vance 2007; Jenkyns 2007; Bebbington 2008.

4 Turner 1981, 187-188.

Lettera del 14 giugno 1831, in Calder et alii 2002, 43-44.

Secondo Momigliano 1955, 224-225, i tedeschi si divisero in due fronti, pro e contro Grote.

Tritle 1999; Whedbee 2004; Liddel 2006; Demetriou 2014, 1-15; Kierstead 2014; Sancho Rocher 2015; 2018,20 22. Sul rapporto con James e John Stuart Mill e gli utilitaristi: Pappè 1979; Preyer 1982; Urbinati 2006, 22-29, 6569, 74-81. Sull'approccio scettico al mito e sulla sofistica: Turner 1981, 83-104, 286-297; Huxley 1996; Kahn 1996.

8 Sulla giustificazione dell'impero marittimo da parte di Grote: Liddel 2009, 17-20; 2014a, 241-243.

$9 \quad$ Sulla vita di Grote: Grote 1873; Bain 1873, 1-170; per altri riferimenti, Chambers 1996.

10 Grote $1873,153$.
} 
Da quel momento visse di rendita, dedito agli studi e ai viaggi, sempre in compagnia della moglie Harriet. È lei, dopo la morte del marito, a raccogliere una serie di ricordi personali sulla vita di Grote, che sono ancora oggi una fonte importante sulla sua vicenda intellettuale. ${ }^{11}$ I ricordi della moglie ripercorrono le tappe essenziali della formazione politica e filosofica negli anni giovanili e la militanza nelle file dei Radicali, i quali nel 1833 ottennero una buona rappresentanza nella House of Commons, dopo il Reform Bill del $1831 .{ }^{12}$ Oltre a Grote, vennero eletti personaggi di rilievo quali John Arthur Roebuck e Sir William Molesworth, ma secondo Harriet il marito era la figura più importante. Ella cita un articolo del Times del 1837, in cui Grote viene considerato molto rispettabile come persona, ma politicamente pericoloso per le istituzioni inglesi. ${ }^{13}$ La carriera politica di Grote, tuttavia, terminò molto presto. I Radicali non erano un vero partito ed erano divisi al loro interno. ${ }^{14}$ Anche per questo nel 1841 Grote non ripresentò la sua candidatura, né lo fece in futuro.

Al nome di Grote sono legate alcune iniziative parlamentari, in particolare le mozioni di riforma del sistema elettorale con l'estensione del suffragio e il voto segreto. ${ }^{15}$ Il suffragio fu ampliato molti anni dopo, nel 1867 (Second Reform Act), mentre nel 1872 fu approvato il voto segreto (Ballot Act) ${ }^{16}$ ma, come Harriet annota, il vecchio Grote aveva ormai maturato una sorta di superiore scetticismo e non nutriva più alcuna fiducia nella politica, che per lui era destinata a rimanere una lotta fra le parti, a prescindere dalle riforme: "Every particular class pursuing its own, the result is a universal struggle for the advantages accruing from party supremacy". ${ }^{17}$ Potrebbe sembrare una completa ritrattazione, ma non lo è: faziosità e instabilità politica sono temi cari a Grote fin dagli anni Trenta, come dimostra la recensione del 1839 all'edizione delle opere di Hobbes a cura di William Molesworth. Ne cito un passaggio:

Now the doctrine of Hobbes, despotic as it may be, is at any rate an equalizing doctrine; not sanctioning the enthronement of any favoured or predominant class to intercept for themselves the rays emanating from the governing power, but enforcing a like claim on the part of every subject to partake in this common benefit. ${ }^{18}$

Grote interpreta Hobbes in senso antioligarchico e afferma che la sottomissione al monarca presuppone una sorta di deliberazione razionale da parte del popolo: "Such a doctrine both supposes and favours the widest diffusion of intelligence

11 Su Harriet Grote: Richardson 2014.

12 Sul radicalismo filosofico e politico: Biagini 1992; Kinzer 2014; Loizides 2014. Sul Reform Bill del 1831, che prevedeva l'estensione della rappresentanza e una ridistribuzione più equa di essa: Woodward 1938, 77-83, 87.

13 "He is a banker of unblemished reputation, and with all the influence over a commercial city derived from that important calling ... He is a very amiable and much respected member of society, an accomplished scholar, a man, moreover, most estimable and exemplary in all the relations of domestic life"; "That hon. Gentleman has made himself the frontispiece of a revolutionary code. He has become the representative and the peculiar organ of whatever is most chimerical in theory, most reckless in experiment, most fatal and revolting in hostility to our national institutions" (Grote 1873, 117-118). Ma sull'effettiva rilevanza politica di Grote (non eccelsa): Kinzer 2014, 36-37.

14 Woodward 1938, 89-91; Kinzer 2014, 40-45.

15 Sull'attività parlamentare di Grote e dei radicali, Bain 1873, 19-65 (e sul Ballot in particolare 19-37).

16 Ensor 1936, 23-24; Cornish et alii 2010, 229-231.

17 Grote 1873, 313. Su questo punto Turner 1981, 226; Chambers 1996, 12-13.

18 Bain 1873, 64. 
among the body of the people", ${ }^{19}$ la stessa intelligenza che si poteva raggiungere col mezzo opposto, cioè con un sistema rappresentativo efficace, in grado di annullare gli interessi di classe quanto una monarchia assoluta. ${ }^{20}$

Nella stessa recensione Grote difende anche la dottrina morale di Hobbes, accettando la tesi che $\mathrm{i}$ concetti di giusto o sbagliato dipendano da "pleasure and pain". ${ }^{21}$ Come è noto, questa è anche la conclusione provvisoria a cui giunge Socrate al termine del Protagora, un dialogo che fu molto caro a Grote e agli utilitaristi inglesi perché poneva su basi empiriche la concezione della democrazia. ${ }^{22}$ Il personaggio di Protagora considera la virtù politica una forma di educazione collettiva, una caratteristica che Grote attribuisce alla civiltà ateniese, profondamente e positivamente influenzata dal pensiero dei sofisti. La rivalutazione della sofistica è uno degli aspetti più originali non solo della History, ${ }^{23}$ ma anche dell'opera successiva, il Plato, pubblicata nel $1865 .^{24}$

Fra il 1841 e il 1842, dopo il ritiro dalla vita politica e prima di dedicarsi interamente alla stesura della History, ${ }^{25}$ Grote fece un viaggio in Italia con la moglie. La descrizione è molto vivida: ${ }^{26} \mathrm{i}$ due coniugi inglesi furono affascinati dai paesaggi, dall'arte, dalle rovine romane e greche e naturalmente apprezzarono il clima. Non visitarono la Sicilia, ma si recarono a Paestum. L'interesse per l'Italia non abbandonò Grote, che molti anni dopo seguì le vicende risorgimentali e si appassionò a Garibaldi. Harriet annota che il marito, nonostante l'entusiasmo per le imprese dei Mille, era scettico sull'Italia a causa della presenza del Papa ${ }^{27}$ Grote era anticlericale e antireligioso. Per lui la religione era una forma di arretratezza morale e intellettuale e impediva lo sviluppo democratico, anche perché condizionava in modo negativo il comportamento della massa, verso la quale tutto sommato Grote si mostrò alquanto diffidente. ${ }^{28}$

Un esempio tratto dalla History: quando parla della vicenda della mutilazione delle Erme e della profanazione dei misteri, Grote ammette 1'irrazionalità del popolo $;{ }^{29}$ allo stesso tempo, però, sottolinea che il comportamento degli ateniesi fu

19 Bain 1873, 66.

20 Bain 1873, 67.

21 Bain 1873, 67.

22 Pl. Prt. 352d-357e. Urbinati 2006, 65-69. Sul tema dell'edonismo socratico nel Protagora: Mann - de Harven 2018.

23 Grote 1888 (VII), 32-80 (48-51, 59-63 sulla figura di Protagora); Sancho Rocher 2015, 110-115.

24 Grote 1865 (II), 29-89 (sul Protagora di Platone); 59-67, 78-89 (sulla dottrina morale espressa da Socrate nel dialogo). Per Grote la visione prettamente utilitarista e individualista di Socrate è corretta e integrata dalla visione comunitaria di Protagora, che addirittura anticipa i contenuti della Repubblica: Grote 1965 (II), 83-84; 1888 (VII), 62. Come ha rilevato Kahn, la lettura grotiana di Platone è "pluralistica", nel senso che Platone non attribuisce il suo pensiero a un unico personaggio, neppure a Socrate (Kahn 1996, 56; ma già Turner 1981, 384414; per una visione d'insieme del Plato: Zuckert 2014). Nella recensione al Plato del 1866, Mill enfatizza la concezione di Protagora, per quanto questi non sia in grado di rispondere alla domanda "che cos'è la virtù" posta da Socrate (Mill 1978, 377-440); per Mill (1978, 389-391) la virtù civica e sociale non ha maestri, l'umanità è il maestro, come nell'Atene democratica descritta da Grote. Sulla recensione di Mill: Turner 1981, 401-402.

25 Bain 1873, 66.

26 Grote $1873,143-152$.

27 Grote $1873,249$.

28 Nel 1848 i Grote seguono le notizie sui moti popolari parigini. Secondo Harriet, George formula severi giudizi sulla "insane multitude" e si dice preoccupato per la sorte della "middle class"; Harriet fa un confronto con la comune parigina del 1870: Grote 1873, 184-185. Per Grote ciò che è irrazionale non solo non è democratico, ma è anche immorale: Turner 1981, 223-225; sul suo ateismo: Gibbins 2014.

29 Sull'intera vicenda giudiziaria: Grote 1888 (VI), 4-50. Egli segue fedelmente la narrazione di Tucidide (Th. 6.27-29, 53, 60-61) e la integra con l'orazione Sui misteri di Andocide (And. Myst.). Sullo scandalo delle Erme 
abbastanza mite, diversamente da quanto si può osservare in altri episodi della storia moderna. Grote menziona la condanna alla tortura e alla pena capitale di Jean-François de la Barre per aver ingiuriato un crocifisso di legno sul ponte di Abbeville nel 1766. Anche in quel caso si immaginarono associazioni sacrileghe e complotti di tutti i tipi e Grote afferma che il caso dimostra "how (as in this mutilation of the Hermae at Athens) the occurrence of one act of sacrilege turns men's imagination, belief, and talk to others real or imaginary". ${ }^{30}$ Per esemplificare il terrore giudiziario dopo la partenza di Alcibiade, Grote cita in nota la Storia della Colonna Infame di Alessandro Manzoni, pubblicata nel 1840, in appendice all'edizione finale dei Promessi Sposi, in cui lo scrittore racconta il processo contro gli untori, accusati di aver diffuso la peste a Milano nel 1630. Grote afferma che, mentre nella Milano cristiana del Seicento la procedura giudiziaria era segreta, ad Atene, una città pagana, i processi erano pubblici. ${ }^{31}$ Sempre in nota egli cita anche i processi tenuti in Inghilterra fra il 1678 e il 1679 per il presunto Popish Plot e conclude così:

Both the English judicature on this occasion, and the Milanese judicature on the occasion adverted to in a previous note, were more corrupted and driven to greater injustice by the reigning prejudice than the purely popular Dikastery of Athens in the affair of Hermae, and of the other profanations. ${ }^{32}$

Questo può bastare per dare un'idea generale della mentalità di Grote e del suo metodo. Egli è un filosofo e un politico radicale, ma conserva anche un approccio realistico di stampo hobbesiano. Nelle note non menziona solo fonti antiche, ma anche costumi, leggi, eventi della storia inglese ed europea dal Medioevo fino ai suoi giorni..$^{33}$ Così egli libera l'antichità greca dall'ambito erudito, nel quale era, ed è, tradizionalmente confinata, e la trasforma in una storia universale. Questa vocazione è sottaciuta ed emerge soprattutto nelle note, che, come vedremo meglio in seguito, costituiscono davvero una specie di sotto-testo, in cui l'autore chiarisce il suo pensiero. Nonostante la mole della documentazione, l'esposizione è fluida, leggera e percorsa da un acume che ancora oggi stupisce, se si pensa al tempo abbastanza breve in cui l'intera opera fu pubblicata. I primi due volumi della History portano la data del 1846. Il terzo e il quarto volume escono nel 1847: un periodo che fra l'altro vede Grote testimone diretto dei conflitti fra i cantoni in Svizzera, dove si era recato nella primavera di quell'anno. La situazione gli ricorda l'antica Grecia, come afferma nella prefazione alle Seven Letters on the Recent Politics of Switzerland. ${ }^{34}$ Intanto sono in lavorazione il quinto e il sesto volume della History, che escono nel dicembre del 1848 (datati 1849). Nel 1850 escono il settimo e l'ottavo volume; nel 1852, il nono e il decimo; nel 1853, l'undicesimo; e infine nel 1856, il dodicesimo e ultimo volume.

e dei misteri: Sartori 1957; Ostwald 1986, 322-333; Furley 1996; Graf 2000; Hornblower 2008, 367-381, 453457; Gagné 2009; Canfora 2011, 199-204, 211-235.

30 Grote 1888 (VI), 11-12 n. 4 (dove cita Voltaire).

31 Grote 1888 (VI), 36-37 n. 2.

32 Grote 1888 (VI), 49-50 n. 1. Sulla comparazione a vantaggio di Atene: Kierstead 2014, 163 e n. 3, $199-200$.

33 Liddel 2014a, 233-243 (sui capitoli della History relativi allo sviluppo della democrazia e dell'impero marittimo).

34 Grote 1847, iv; Grote 1873, 175. 


\section{Schömann vs Grote}

La storia di Grote è atenocentrica e considera la fondazione e il perfezionamento della democrazia come l'aspetto più innovativo della civiltà greca. In questo egli si contrappose alla lettura conservatrice (ma non reazionaria) di William Mitford, ${ }^{35}$ completando una riabilitazione già iniziata dalla History of Greece di Connop Thirlwall. ${ }^{36}$ Secondo Grote, la democrazia ateniese aveva una funzione educativa, insegnava al popolo a governare, lo rendeva sempre più attivo, unito e cosciente del suo ruolo $; 37$ non solo, i cittadini avevano acquisito un senso morale e politico che prima non possedevano, un senso di appartenenza e un rispetto della legge che non furono facili da ottenere, ma una volta raggiunti produssero un governo stabile, come quello inglese e americano. Il passo è noto, ma è opportuno citarlo:

This co-existence of freedom and self-imposed restraint -of obedience to authority with unmeasured censure of the persons exercising it- may be found in the aristocracy of England (since about 1688) as well as in the democracy of the American United States: and because we are familiar with it, we are apt to suppose it a natural sentiment; though there seem to be few sentiments more difficult to establish and diffuse among a community, judging by the experience of history. We may see how imperfectly it exists at this day in the Swiss Cantons; while the many violences of the first French Revolution illustrate, among various other lessons, the fatal effects arising from its absence, even among a people high in the scale of intelligence. Yet the diffusion of such constitutional morality, not merely among the majority of any community, but throughout the whole, is the indispensable condition of a government at once free and peaceable; since even any powerful and obstinate minority may render the working of free institutions impracticable, without being strong enough to conquer ascendency for themselves. ${ }^{38}$

Nel sottolineare l'educazione civica Grote è visibilmente influenzato dal mito delle origini dell'uomo narrato da Protagora nell'omonimo dialogo platonico, in cui la virtù politica non è innata ma può essere insegnata ai cittadini all'interno della comunità con la scuola e le leggi. ${ }^{39}$ Grote riconosce nella storia un meccanismo evolutivo, che conduce l'umanità verso forme di convivenza sempre migliori. Questo è il motivo per cui egli pone il sorteggio delle cariche molti anni dopo la riforma clistenica, in quanto lo ritiene più consono a una democrazia più matura, in un'epoca in cui l'accesso alle magistrature fu allargato anche alle altre classi di censo. ${ }^{40}$ Egli è

35 La storia di Mitford viene recensita da Grote nel 1826: Bain 1873, 12-18. Già qui Grote mette in rilievo l'importanza della democrazia per lo sviluppo del pensiero e della civiltà. Sul conservatorismo di Mitford: Turner 1981, 192-204

36 Turner 1981, 211-213. Importante, in tal senso, anche l'opera di E. Bulwer Lytton (Athens: Its Rise and Fall): si veda Murray 2004, 15-34. Sul tema (con ulteriore bibliografia) si veda inoltre Canevaro c.s.

37 Grote 1888 (III), 394-398.

38 Grote 1888 (III), 372-373.

39 Pl. Prt. 323c-328c. Grote 1865 (II), 45-47, e 46 n. K, in cui Grote nota la coincidenza fra Protagora e James Mill su questo tema. Sulla combinazione fra diritti e doveri nell'Atene di Grote: Liddel 2006, 155-157. Sul "carattere" di un popolo (come insieme di qualità mentali e morali) in età vittoriana e, di conseguenza, in Grote: Kierstead 2014, 182-190. Sul concetto di "moralità costituzionale": Sancho Rocher 2015, 101-110.

40 Grote 1888 (III), 361-368: sulla base di Plu. Arist. 22.1, al quale oggi non si crede più, perché si segue Arist. Ath. 8.1 (Solone introduce il sorteggio fra i candidati all'arcontato), 22.5 (reintroduzione del sorteggio nel 487/6 
convinto che "the lot was a symptom of pronounced democratical spirit, such as we must not seek in the Solonian institutions". ${ }^{41}$

Le critiche a questa visione positiva però non mancarono. Nel 1854, Georg Friedrich Schömann pubblicò un saggio interamente dedicato alla confutazione delle tesi di Grote (Die Verfassungeschichte Athen's nach G. Grote's History of Greece kritisch geprüftt). ${ }^{42}$ Schömann contestava l'impostazione evoluzionista dello storico inglese e tendeva a retrodatare una serie di istituti, oltre a dissentire su questioni più specifiche come la fondazione e il numero dei demi. ${ }^{43}$ In particolare Schömann attribuiva il sorteggio degli arconti a Clistene, che avrebbe agito così non per garantire l'uguaglianza fra i cittadini, ma per impedire al rivale Isagora di influenzare il popolo nelle elezioni ed eleggere i suoi candidati. Anche questo passo merita di essere citato per esteso, perché è l'esatto rovesciamento di quanto dice Grote:

Such intrigues Kleisthenes thought it his duty to provide against. Therefore he abolished popular elections, and introduced selection by lot for a great part of the offices; believing that this would secure appointments in most cases no worse, and in many much better, than those made by the votes of a populace misled by faction and intrigue. I should have thought that even in England there had been enough opportunity to judge of the value of this kind of popular election. At least, here in Germany we have had experience which would justify us in concluding, that it was impossible to make worse appointments by the chance of the lot than by the votes of the masses, guided by demagogues and party-leaders. ${ }^{44}$

Per Schömann il voto popolare non era in grado di emancipare la massa né produceva scelte migliori del sorteggio, perché era necessariamente condizionato dai "party-leaders", e le elezioni non servivano a scegliere i più esperti, anzi potevano avere un esito peggiore di un'estrazione a sorte. ${ }^{45}$ È evidente che il suo obbiettivo

a.C.) e 26.2 (estensione agli zeugiti della candidatura all'arcontato nel 457/6 a.C.). Su questi passi: Rhodes 1992, 146-149, 272-275, 328-331.

41 Grote 1888 (II), 499.

42 Schömann 1854. Nel 1878 l'opuscolo fu pubblicato in traduzione inglese da Bernard Bosanquet: Schömann 1878 .

43 Sui demi: Schömann 1878, 64-68 (i demi erano solo cento, sulla base di una interpretazione errata di Hdt. 5.69); Grote 1888 (III), 315-353 e 351 n. 1 (i demi erano più di cento). Sull'eliea: Schömann 1878, 38-40 (per lui è un tribunale separato dall'assemblea, già prima di Clistene); Grote 1888 (III), 359-361 (in età arcaica l'eliea corrisponde all'assemblea popolare). Sui nomoteti Schömann 1878, 56-64 (forse già istituiti da Solone); Grote 1888 (IV), 456-458 (introdotti in età periclea). Sul sorteggio degli arconti: Schömann 1878, 73-82 (già introdotto da Clistene per impedire a Isagora di influenzare le elezioni popolari); Grote 1888 (III), 362-368 (introdotto solo dopo la vittoria a Platea contro i persiani). Qualche riferimento bibliografico essenziale: sui demi Traill 1975; 1986; Whitehead 1986; sull'evoluzione dei tribunali Boegehold et alii 1995; sui nomoteti Hansen 1991, 205212; Canevaro 2018. Sul sorteggio dei magistrati Hansen 1990. L'opuscolo su Grote di Schömann anticipò la pubblicazione, avvenuta l'anno dopo, della sua opera più significativa, i Griechische Alterthümer: Schömann 1855. L'opera ebbe varie edizioni: l'ultima, postuma, fu revisionata da Lipsius (Schömann 1897). Sul duello Grote-Schömann: Marcaccini 2012, 79-82.

44 Schömann 1878, 81.

45 Nei Griechische Alterthümer 1'autore sostiene un concetto analogo: "Many indeed have found it utterly incredible that such a mode of filling up offices, which seems to them adapted only to the most absolute democracy, can have been introduced so early as the reforms of Clisthenes. We have, however, already remarked that the institution of the lot must not always be regarded as a proof of democratic absence of restraint, but that it was adopted as a means of avoiding the intrigues of party contests which occur only too easily at popular 
non era soltanto contestare l'immagine positiva della democrazia ateniese, come apice dello sviluppo civile dei greci, ma l'idea della democrazia tout court. Grote e Schömann non offrono solo diverse ricostruzioni del passato, ma hanno concezioni antropologiche opposte. L'unica cosa che li accomunava era l'ignoranza dell'Athenaion politeia di Aristotele, scoperta e pubblicata alla fine dell'Ottocento: ${ }^{46}$ una lacuna che vizia le loro opere in molti punti.

\section{I tribunali popolari}

Nei capitoli 44-47 della History Grote intreccia l'evoluzione della democrazia sotto Pericle con la costruzione dell'impero. ${ }^{47} \mathrm{Nel}$ cap. 44 egli parla degli avvenimenti immediatamente successivi alla seconda guerra persiana, della fondazione della lega di Delo e delle riforme democratiche di Aristide. Nel cap. 45 tratta dell'espansione dell'impero marittimo ateniese e giunge fino alla pace trentennale con Sparta, che egli data al 445 a.C. Il cap. 46 è interamente dedicato alla riforma giudiziaria di Efialte e Pericle, mentre il cap. 47 ripercorre gli eventi fino alle premesse della guerra del Peloponneso e affronta la questione dell'imperialismo ateniese e dei rapporti con gli alleati. Nonostante l'accento sulle riforme nel cap. 46, Grote sembra dare la precedenza alla politica estera, che ritorna anche nel cap. 47. Ci si può chiedere se per Grote sia stata la democrazia a favorire la costruzione dell'impero o, viceversa, sia stato l'impero a rendere possibile la democrazia ${ }^{48}$ ma egli non si preoccupa di stabilire un nesso diretto né in un senso né nell'altro. Il suo approccio è empirico. Quando parla delle riforme attuate da Aristide dopo Salamina, ${ }^{49}$ le considera come una conseguenza della vittoria, ma allo stesso tempo afferma che Atene non è giunta all'impero con un intento deliberato, ma grazie alla fortuna e per progressivi aggiustamenti in base alle esigenze imposte dai tempi. L'atto della fondazione della lega fu favorito dall'incapacità spartana, ${ }^{50}$ dalla casuale presenza di Aristide in Ionia ${ }^{51}$ e dalla paura delle città ioniche, che accettarono di buon grado l'egemonia ateniese, ${ }^{52}$ mentre il passaggio dall' egemonia all'impero avvenne in modo graduale, nel periodo che precedette la pace trentennale del 445 a.C., senza che si possa stabilire un momento preciso $^{53}$ e soprattutto grazie alla cessione spontanea della forza militare da

elections" (Schömann 1855, 339). Queste parole vengono tagliate in Schömann 1897, 354, dove Lipsius aggiunge che tre anni dopo Maratona fu reintrodotto il sorteggio come al tempo di Solone, sulla base di Aristotele (vedere n. 40).

46 Kenyon 1891. Mirko Canevaro mi ha gentilmente segnalato che Schömann continuava a basarsi sul De republica atheniensium di Sigonio, un'opera del XVI secolo.

47 La numerazione dei capitoli non cambia nelle varie edizioni: nell'edizione postuma del 1888 essi sono compresi nel IV volume.

48 L'equazione navy-democracy è antica: ad esempio [X.] Ath. 1.2; Arist. Pol. 1274a 13-15. Su questa linea: Strauss 1996; Raaflaub 2007, 121-136; Tritle 2013. Viceversa, la democrazia è una condicio sine qua non dell'impero per Ober 1996, 53-71; 2007, 100. In particolare contro questa idea Samons 2010, 28-31, mentre per una visione critica del nesso guerra-società Rhodes 2007, 30; van Wees 2007, 295-298.

49 Vedere n. 40.

50 Grote 1888 (IV), 350-351.

51 Grote 1888 (IV), 353.

52 "It was their common fear, rather than Athenian ambition, which gave birth to the alliance; and they were grateful to Athens for organizing it" (Grote 1888 (IV), 355).

53 Grote 1888 (IV), 381. 
parte degli alleati, esattamente come accadde in alcune parti dell'India, in cui alcuni principi cedettero il loro potere agli inglesi. ${ }^{54}$

Come Tucidide e Plutarco, ${ }^{55}$ Grote contrappone la solerzia ateniese alla pigrizia degli alleati e afferma che sarebbe stato contrario alla "moralità pubblica" rinunciare alla supremazia politica, militare ed economica sull'Egeo: "it would have required a more self-denying public morality than has ever been practised by any state, either ancient or modern, even to conceive the idea of relinquishing voluntarily an immense ascendency as well as a lucrative revenue". ${ }^{56}$ L'impero fu la giusta ricompensa delle grandi capacità degli ateniesi: era nella natura delle cose che la confederazione si mutasse in una dominazione dello stato più forte su quelli più deboli, poiché era impossibile mantenere un rapporto paritario con gli alleati. ${ }^{57} \mathrm{La}$ loro irrilevanza in quanto tributari, e quindi la loro sudditanza, è confermata dal trasferimento del tesoro della lega da Delo ad Atene, che non fu un atto di forza, ma fu proposto da Samo. ${ }^{58}$ L'impero è dunque una combinazione di fattori, fra i quali, come vedremo, va contata anche la democrazia radicale inaugurata da Efialte e Pericle, che ha senz'altro consentito una gestione migliore del potere.

Grote descrive l'istituzione dei tribunali popolari nel cap. 46. ${ }^{59}$ Allo stesso complesso di iniziative vengono associati anche il sorteggio dei magistrati e il pagamento delle giurie, l'introduzione dei nomophylakes e dei nomoteti, la graphe paranomon: ${ }^{60}$ tutte questioni sulle quali si concentra la polemica di Schömann, come abbiamo già visto. ${ }^{61}$ La caratteristica generale che Grote riconosce ai nuovi tribunali è l'incorruttibilità e la capacità di punire anche i ricchi e i potenti. ${ }^{62}$ Il caso di Anito, che comprò la giuria e si fece assolvere, sembrerebbe provare la tesi contraria, ovvero il nesso fra la corruzione e l'estrazione popolare dei giudici, come sostiene Aristotele nella Costituzione degli ateniesi ${ }^{63}$ Tuttavia Grote rileva che Anito corruppe la giuria poco prima della fine della guerra, ${ }^{64}$ quando lo stato non aveva niente in cassa e spendeva tutto per la guerra; così anche i tribunali, forse convocati più raramente, $\mathrm{e}$

54 "The military force of these subject-states was thus in a great degree transferred to Athens by their own act, just as that of so many of the native princes in India has been made over to the English" (Grote 1888 (IV), 387-388). Su questo e altri passi, in cui Grote giustifica l'impero: Liddel 2014a, 241-242.

55 Th. 1.99, 1.70.2-9; Plu. Cim. 11.

56 Grote 1888 (IV), 389.

57 "Amidst such glaring inequalities of merit, capacity, and power, to mantain a confederacy of equal members was impossible. It was in the nature of things that the confederacy should either break up, or be transmuted into an Athenian empire" (Grote 1888 (IV), 390-391).

58 Plu. Arist. 25.3; Per. 12.1-2. Grote (1888 (IV), 428-429) colloca l'evento nel 449 a.C. In realtà il passaggio sembra avvenire nel 454/3 a.C., dopo la fine della campagna militare in Egitto, come si può dedurre dalle liste delle sessagesime versate nel tesoro di Atena ( $I G \mathrm{I}^{3}$ 259: prima lista delle aparchai): si veda innanzitutto Samons 2000, 92-106, per un vaglio critico delle testimonianze letterarie ed epigrafiche; cf. inoltre Meiggs 1972, 109128, 538-561 (tavole dei pagamenti dal 453 al 420 a.C.); Rhodes 1985, 15; McGregor 1987, 59-61; Figueira 1998, 265-295; Constantakopoulou 2007, 69-75. Grote venne a conoscenza delle liste delle aparchai dalla seconda edizione (1851) della Staatshaushaltung der Athener di August Boeckh, ma non ne tenne conto: Liddel $2009,24$.

59 Grote 1888 (IV), 438-486.

60 Rispettivamente: Grote 1888 (IV), 441-443, 455-458, 459-63. Sui nomophylakes: Bearzot 2007; sulla graphe paranomon: Hansen 2001

${ }_{61}$ Inutile entrare nei dettagli della riforma, sui quali l'analisi di Grote è inevitabilmente datata. Sulle giurie popolari a partire dal 462 a.C.: Harrison 1971, 239-241; Boegehold et alii 1995, 23-30.

62 Grote 1888 (IV), 465-469.

63 Arist. Ath. 27.4-5.

${ }_{64}$ Come apprende da Plu. Cor. 9. 
senza certezza della paga, potevano essere composti in modo arbitrario e integrati con giudici corruttibili, cosa che prima non avveniva. ${ }^{65}$ Sono considerazioni molto interessanti, se pensiamo che in effetti non è ben chiaro quale fosse il regime vigente ad Atene dopo la caduta del governo oligarchico dei Quattrocento. Sappiamo che nel 410 a.C. il potere passò ai Cinquemila, ${ }^{66}$ ma nessuna fonte parla di una nuova transizione alla democrazia radicale ${ }^{67}$ e c'è chi ha messo in dubbio che essa sia stata effettivamente restaurata prima della fine dell'oligarchia dei Trenta. ${ }^{68}$

Le giurie popolari diedero al popolo ateniese il potere di inibire la deriva oligarchica che, nell'ottica di Grote, corrisponde alla prevalenza di un interesse particolare su quello generale. Per spiegare cosa intende, egli ricorre come al solito alla storia moderna. In nota scrive che la Camera stellata (Star Chamber) fu istituita alla fine del Quattrocento "in order "to deal with offenders too stout for the ordinary course of the justice"; 69 allo stesso modo nel periodo che va da Enrico III a Enrico VIII "laws were enacted for restraining the feuds, robberies, and oppressions of the barons and their dependents on the one side, and to moderate and check the excesses and extortions of the royal purveyors on the other; these being the two capital evils then felt". ${ }^{70}$ Grote menziona di nuovo i Promessi Sposi di Alessandro Manzoni, in quanto il romanzo "exhibits a state of judicial administration, very similar to that above described, in the Milanese, during the sixteenth and seventeenth centuries; demonstrated by repeated edicts, all ineffectual, to bring powerful men under the real control of the laws". ${ }^{71}$ Assolutismo e democrazia sono evidentemente accomunati dall'avversione al potere oligarchico, che Grote considera il peggiore dei mali. Per lui il modello ateniese e quello inglese dopo il 1688 si somigliano, ma il sistema ateniese, a differenza di quello inglese, metteva in pratica la teoria; gli ateniesi ricorrevano a un certo numero di privati cittadini, nominati a sorte, obbligati sotto giuramento ad ascoltare senza pregiudizi accusa e difesa e tenuti ad emanare un verdetto sulla base della loro coscienza, "but in Athens this theory was worked out to its natural consequences; while English practice, in this respect as in so many others, is at variance with English theory" ${ }^{72}$ Grote ribadisce il concetto più volte: i giuristi che elogiano l'amministrazione della giustizia in Inghilterra e negli Stati

65 Grote 1888 (IV), 468-9 n. 2 ; 1888 (VI), 301-308 (sulla transizione dai Cinquemila alla piena democrazia dopo il 411/10 a.C.; Grote sottolinea che si tratta di un periodo di grave deficit finanziario).

66 Th. 8.97.1-2; Arist. Ath. 33.2.

67 In Arist. Ath. 41.2 c'è un elenco delle costituzioni ateniesi dalle origini al 401 a.C.: l'ottava è l'oligarchia dei Quattrocento, la nona è di nuovo la democrazia, senza alcun accenno ai Cinquemila. Due le ipotesi: o Aristotele non si è curato di nominarli, oppure essi potrebbero coincidere con la democrazia restaurata dopo la caduta dei Quattrocento. In questa ottica i Cinquemila non sarebbero un regime oligarchico transitorio, ma una democrazia più moderata che consentiva l'accesso alle magistrature a un numero limitato di cittadini (cinquemila appunto), ma garantiva a tutti i cittadini la partecipazione all'assemblea e ai tribunali. Questa è la tesi di de Ste. Croix 1956; contrario Rhodes 1972. Per altra bibliografia sui Quattrocento e i Cinquemila rimando a Marcaccini 2013; 2017.

68 Sealey 1975 (a favore di una graduale transizione dai Cinquemila alla democrazia negli anni successivi al 410 a.C.); Gallucci 1986.

69 Grote 1888 (IV), 466 n. 1, cita Thomas Smith, Treatise on the Commonwealth of England (in realtà De Republica Anglorum. The Maner of Governement or Policie of the Realme of England, pubblicato a Londra nel 1583). Grote menziona anche gli Hybristodikai di Eupoli, una commedia perduta su "quelli che non vogliono farsi processare" (Kassel - Austin 1988, 466; Grote si basa su Meineke 1839, 145). Sulla Camera stellata vedere Baker 2003, 195-200.

70 Grote 1888 (IV), 466 n. 1 cita Josiah Tucker, A Treatise concerning Civil Government, pubblicato a Londra nel 1781.

71 Grote 1888 (IV), 467 n. 1.

72 Grote 1888 (IV), 469-470. 
Uniti hanno in mente i tribunali ateniesi, poiché ad essi si ispira il sistema moderno delle giurie popolari, senza che però ne venga veramente attuato lo spirito. ${ }^{73}$

Riassumendo, le riforme di Pericle sono presentate come il rimedio più efficace contro l'oligarchia in quanto conferiscono ai tribunali popolari una sorta di sovranità assoluta fondata sulla legge e capace di garantire l'uguaglianza e l'interesse comune contro gli interessi di parte. Come nell'Inghilterra moderna il potere giudiziario del re si articolava in una serie di corti col fine di dispensare una giustizia imparziale, anche contro funzionari delegati dallo stesso potere regio, così ad Atene, in un'epoca in cui l'amministrazione si faceva sempre più complessa, per il potere e le ricchezze che aumentavano grazie all'impero marittimo, i tribunali erano chiamati a ristabilire l'equilibrio e la coesione all'interno della comunità: nell'ottica di Grote assolutismo e democrazia non solo hanno l'oligarchia come nemico comune, ma sembrano condividere il medesimo principio di sovranità.

\section{Government of dependencies}

Veniamo ora all'intersezione fra politica interna e politica estera. Il capitolo $47,{ }^{74} \mathrm{che}$ chiude la parte dedicata alla Pentecontetia, mostra come il sistema giudiziario pericleo abbia svolto una funzione importante anche nella gestione dell'impero. ${ }^{75} \mathrm{In}$ queste pagine passato e presente sono quasi sovrapposti, ma giungono addirittura a fondersi alla fine del capitolo, in una lunga nota. ${ }^{76}$ Qui Grote esemplifica il ruolo di garanzia dei tribunali popolari ateniesi con un caso giudiziario del 1773, in cui un suddito di Minorca riuscì a far valere i suoi diritti contro il governatore Mostyn grazie a un tribunale inglese. La sua fonte è un saggio di George Cornewall Lewis, intitolato Essay on the Government of Dependencies, dove l'autore aveva già classificato in questo modo l'impero ateniese e quello britannico. Scrive Grote:

It is to be recollected that the Athenian empire was essentially a government of dependencies; Athens as an imperial state exercising authority over subordinate governments. To maintain beneficial relation between two government -one supreme, the other subordinate- and to make the system work to the satisfaction of the people in the one as well as of the people in the other, has always been found a problem of great difficulty. Whoever reads the instructive volume of Sir G.C. Lewis ... and the number of instances of practical misgovernment in this matter which are set forth therein, will be inclined to think that the empire of Athens over her allies makes comparatively a creditable figure. ${ }^{77}$

Grote aveva un'alta considerazione di Lewis e del suo lavoro. Perciò, prima di ritornare alla History, è opportuno soffermarsi sulla figura e sull'opera di Lewis. ${ }^{78}$ Questi non solo fu uno degli amici più stretti di Grote, ma anche un pregevole

\footnotetext{
73 Grote 1888 (IV), 471-472 e 472-474 n. 1. Grote ammette anche i difetti del sistema antico, che sono l'assenza di una procedura precisa e di un giudice competente come arbitro: 1888 (IV), 473-480.

74 Grote 1888 (IV), 487-557.

75 Sulla giurisdizione imperiale: de Ste. Croix 1961; Meiggs 1972, 220-233; Liddel 2010; Low 2013.

76 Grote 1888 (IV), 531-533 n. 3.

77 Grote 1888 (IV), 531-532 n. 3.

78 Sulla biografia e la bibliografia di Lewis: Calder et alii 2002, xi-xvii.
} 
studioso di antichità, "uno dei maggiori, e forse il più acuto e il meno subalterno, divulgatore dell'Altertumswissenschaft tedesca in Inghilterra": ${ }^{79}$ nel 1828 pubblicò la traduzione della Staatshaushaltung der Athener di August Boeckh ${ }^{80}$ che Grote cita più volte; nel 1831 uscì la sua traduzione dei Dorier di K. O. Müller e nel 1840 l'edizione inglese della History of the Literature of Ancient Greece to the Period of Isocrates dello stesso Müller, col quale Lewis fu in corrispondenza dal 1828 al $1839 .{ }^{81}$ La sua opera più importante è dedicata alla storia romana (An Inquiry into the Credibility of the Early Roman History, London 1855), in cui polemizza col metodo seguito da Barthold Georg Niebuhr, mentre nel 1863, l'anno della morte, esce il Dialogue on the Best Form of Government, in cui mette a confronto democrazia, oligarchia e monarchia, come nel dialogo fra Otane, Megabizo e Dario nel terzo libro di Erodoto (3.80-83). Lewis fu anche membro del Partito Liberale e rivestì importanti incarichi di governo: all'apice della carriera fu Chancellor of the Exchequer nel primo governo Palmerston dal 1855 al 1859, e Home Secretary e poi Secretary for War nel secondo governo Palmerston dal 1859 al $1863 .^{82}$ L'amicizia fra lui e Grote risale al 1835, come testimonia la moglie Harriet: "The intimacy with Mr. George Cornewall Lewis ... had begun about the year 1835 ... Both of them devoted to learned studies, and mutually attracted by certain affinities of intellectual character, the two scholars became and continued steadfast friends through life". ${ }^{83}$ Quando Lewis morì nell'aprile del 1863, Grote subì un duro colpo: "His sorrow was indeed poignant, for the void left in his personal sympathies and familiar commerce of thought by his beloved friend's loss, was irreparable". ${ }^{84}$

Nell'Essay on the Government of Dependencies del 1841 Lewis aveva ben identificato le analogie fra il dominio marittimo ateniese e quello britannico, individuando nella giurisdizione uno dei tratti caratteristici del rapporto fra il governo dominante $\mathrm{e} i$ governi subalterni. La dependency è una comunità politica soggetta $\mathrm{a}$ un governo subordinato, mentre la comunità che fa parte del governo supremo, al quale è sottoposta la dependency, è definita "the dominant community, or country". 85 Questa è appunto Atene al tempo dell'impero, quando gli ateniesi stessi chiamavano

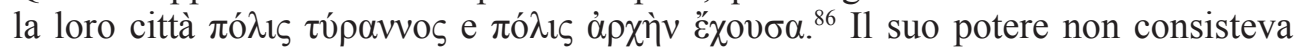
tanto nell'esazione del tributo o nella forza militare, che contraddistinsero la prima fase della lega di Delo, quando gli alleati erano, almeno formalmente, in una condizione di parità con Atene, ma soprattutto nell'invadenza negli affari interni della comunità dipendente, che segnò il passaggio dalla lega all'impero. ${ }^{87}$ Lewis era stato influenzato da Boeckh, che aveva posto la giurisdizione come un elemento fondamentale per distinguere fra alleati indipendenti e sudditi. Secondo Boeckh

\footnotetext{
79 Canfora, in Lewis 1996, 12.

80 Boeckh 1842.

81 Fu Lewis a proporre a Müller di scrivere una storia della letteratura greca e di pubblicarla in Inghilterra: lettere del 24 maggio e del 4 luglio 1834, in Calder et alii 2002, 73-77.

82 Woodward 1938, 640-641.

83 Grote 1873,126

84 Grote $1873,266$.

85 Lewis $1841,70$.

86 In primo luogo Lewis 1841, 70 (in nota) menziona Th. 1.122.3, 124.3, 6.85.1; e poi, per dimostrare che la parola $\grave{\alpha} \rho \chi \eta ́$ si addice a un governo delle dipendenze, cita anche Th. 1.67.4 (in relazione al decreto megarese), 2.97.1 (sul regno degli Odrisi), 3.37.2 (discorso di Cleone contro i Mitilenesi), 6.90 .3 (discorso di Alcibiade presso 1'assemblea degli spartani).

87 Lewis 1841, 102-106.
} 
(nella traduzione di Lewis) "the subject allies were compelled to try all their disputes in the courts of Athens". ${ }^{88}$

Per dimostrare questa tesi Lewis passa in rassegna anche altre realtà storiche, quali Cartagine, Roma, la Spagna, la Francia, le repubbliche marinare italiane, in particolare Venezia, e infine l'Inghilterra a partire da Elisabetta I. ${ }^{89}$ L'impero britannico in linea di massima rispettava le usanze delle colonie, ma si poneva il problema della compatibilità fra le leggi inglesi e quelle locali. Uno dei casi più significativi è quello menzionato da Grote, cioè il processo Fabrigas vs Mostyn, discusso presso la Court of Common Pleas nel 1773. ${ }^{90}$ Dagli atti si apprende che Mostyn aveva arrestato Fabrigas e lo aveva deportato in Spagna senza processo, poiché questi aveva presentato una richiesta di risarcimento che al governatore sembrava illegittima. Mr. Justice Gould, che trattò la causa presso la Corte, lasciò alla giuria decidere se Fabrigas avesse avuto un comportamento sedizioso o se avesse chiesto solo un rimborso per aver subito dei danni, e la giuria decise di dargli ragione stabilendo un risarcimento di 3.000 sterline. In seguito fu fatta domanda per un nuovo processo, ma la Corte rifiutò. Lord C. J. De Grey motivò il rifiuto sostenendo che Mostyn aveva bandito Fabrigas sulla base di un vecchio ordinamento di Minorca, in cui era prevista anche la tortura, ma siccome le leggi inglesi vietavano sia il bando sia la tortura, in tal caso esse dovevano prevalere su quelle della comunità dipendente. Va osservato anche che la Corte considera il governatore di Minorca privo di potere legislativo, in virtù del fatto che questi è solo un king's servant. A questo proposito Lord Mansfield, membro della Corte reale (King's Bench), presso la quale Mostyn aveva presentato un'istanza di revisione (Writ of Error), si scandalizzò che un governatore potesse agire a suo piacimento, senza rispondere dei suoi atti in un tribunale. $^{91}$

Lewis fa sentire anche l'altra campana, trascrivendo una parte dell'arringa del difensore di Mostyn, di cui non fa il nome, il quale affermò che una dependency andava governata solo nell'interesse dello stato dominante e che gli inglesi dovevano scoraggiare il patriottismo all'estero se volevano tenersi le colonie: “... they will take care of all our conquests abroad. If that spirit [sc. il patriottismo] prevailed in Minorca, the consequence of it would be the loss of that country, and of course our Mediterranean trade". ${ }^{22}$ Queste parole colgono il vero nocciolo della questione, come dimostra un secondo caso esposto da Lewis. ${ }^{93}$ Stavolta il processo è contro il governatore inglese di Trinidad, Thomas Picton, che nel 1801 aveva torturato Luisa Calderon per estorcerle una confessione. La domanda che si pongono i giuristi è la

88 Boeckh 1842 (III), 403. Contro questa distinzione de Ste. Croix 2008, 249-254.

89 Lewis 1841, 110-166. Lewis affronta il problema giurisdizionale soprattutto nel cap. 5 del saggio (1841, 189210), ma la questione ritorna più volte anche in altre parti: 1841, 244-246 (vantaggi del paese subordinato se il paese dominante abolisce la schiavitù, come nelle Indie occidentali britanniche); 1841, 252-286 (svantaggi del paese subordinato se il paese dominante tende ad avocare a sé la giurisdizione senza tenere conto delle usanze e delle leggi locali); 1841, 294-296 (conseguenze positive, in caso di malgoverno, della forma popolare del governo dominante, presso il quale un membro del potere legislativo può diventare portavoce delle istanze del popolo sottomesso; ma conseguenze negative se il governo locale si dota esso stesso di un sistema di rappresentanza e, teoricamente, governa in modo autonomo).

90 Lewis $1841,367-369$. Il caso è esposto in una delle note finali del volume, la nota M (366-374), in cui l'autore esamina anche altri processi simili. Sulla Court of Common Pleas e le sue competenze: Baker 2003, 125-143.

91 Lewis 1841, 368-369. Sulla Court of King's Bench: Baker 2003, 145-158.

92 Lewis $1841,368$.

93 Lewis $1841,369-374$. 
stessa del caso Fabrigas: per quanto la legge di Trinidad approvasse la tortura, era il governatore tenuto ad applicarla? Vi furono vari processi presso la Corte reale, in cui fu formata una speciale giuria presieduta da Lord Ellenborough, ma non si giunse mai a una sentenza definitiva. Rimangono i verbali delle discussioni, dai quali emergono pareri diversi: per esempio, Mr. Nolan sostenne che la legge locale era valida solo se non era contraria a quella inglese e che in tal caso il governatore aveva sbagliato nell'applicarla, ma Lord Ellenborough sollevò un'obiezione di fondo: dato che il governatore, delegato dal re, aveva ordinato la tortura secondo le leggi di Trinidad, si doveva supporre che lo stesso re avesse compiuto un atto illegittimo? In altre parole Ellenborough si chiedeva se in situazioni del genere il re avrebbe dovuto essere limitato dalla legge. In caso di risposta affermativa, la giurisdizione britannica si sarebbe estesa di fatto in tutte le colonie e le dependencies avrebbero perso la loro autonomia, per quanto parziale, diventando parte integrante del paese dominante, e questo non sarebbe stato conveniente in termini economici. Lord Ellenborough evidenziò la difficoltà di individuare un confine netto fra leggi inglesi e leggi locali e concluse che queste ultime sarebbero dovute rimanere in vigore finché lo stato dominante non le avesse cambiate.

Lewis non prende una posizione esplicita su questi pareri, ma chiarisce un punto importante: laddove lo stato dominante tendesse ad avocare interamente l'amministrazione della giustizia, la dependency scomparirebbe in quanto sarebbe di fatto assorbita dallo stato dominante $;{ }^{94} \mathrm{~d}$ 'altra parte la dependency non può essere del tutto autosufficiente nel campo della giustizia, come non può dotarsi di un sistema di rappresentanza autonomo, poiché anche in questo caso essa cesserebbe di esistere e diverrebbe uno stato indipendente. ${ }^{95}$ L'impero britannico poteva essere definito un government of dependencies per il fatto che esso garantiva una certa autonomia interna alle colonie, senza affrontare il problema della giurisdizione in modo sistematico, adottando soluzioni diverse a seconda dei casi. ${ }^{96}$

Torniamo a Grote e cerchiamo di capire se egli ha una visione analoga a questa. Grote rifiuta il giudizio negativo del Vecchio Oligarca, secondo il quale i sudditi erano schiavi dell'intero popolo ateniese assiso nei tribunali, ${ }^{97}$ ma prende per buono quanto dice l'ambasciatore ateniese a Sparta nel 432 a.C., per il quale le giurie popolari erano tanto imparziali che gli ateniesi si mettevano in una condizione di inferiorità. ${ }^{98}$ Il passo

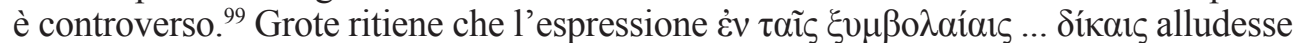
solo ai processi che concernevano i sudditi e che venivano celebrati ad Atene, ed

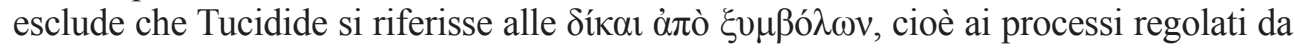

94 Lewis $1841,105-106,325-341,381-382$ n. Q.

95 Lewis 1841, 295-296.

96 Cf. Burroughs 1999; Porter 1999.

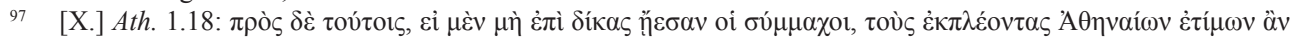

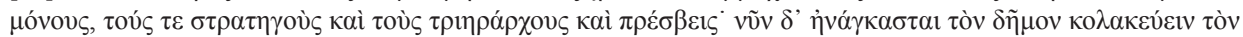

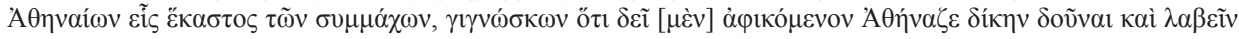

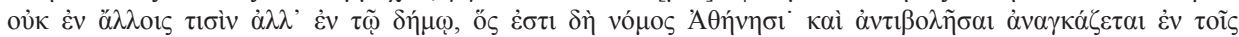

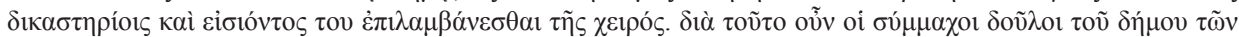

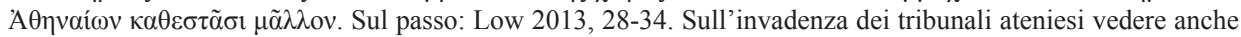
Antiph. 5.47 (che riguarda però solo le condanne a morte: de Ste. Croix 1961, 271).

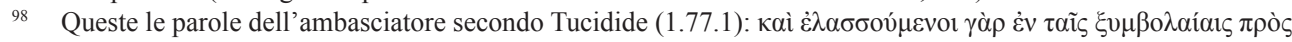

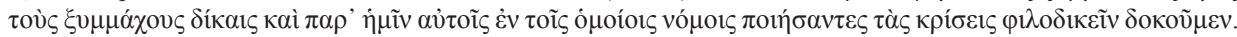

99 Hopper 1943, 35-36; de Ste. Croix 1961, 96-100; Meiggs 1972, 228-233; Fornara 1979, 52 n. 2; Cataldi 1983, 231-250; Hornblower 1991, 122-123; Pébarthe 2007, 247-249. 
un trattato interstatale, che potevano svolgersi anche fuori da Atene. ${ }^{100}$ Addirittura per

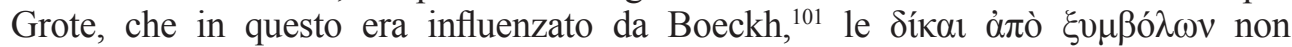
riguardavano mai gli stati sudditi. Oggi invece questa ipotesi è superata, per quanto sul resto del passo le interpretazioni siano diverse. Per me la lettura più convincente è quella di Meiggs, che vede in Tucidide due diverse fattispecie giudiziarie: le controversie fra Atene e gli alleati, che potevano essere discusse anche in tribunali stranieri (nei quali gli ateniesi erano penalizzati: $\dot{\varepsilon} \lambda \alpha \sigma \sigma o v ́ \mu \varepsilon v o \imath)$ e il trasferimento di alcuni processi

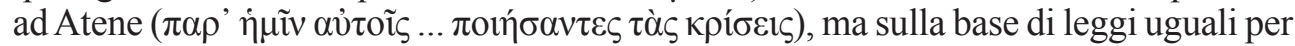

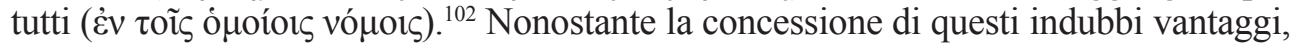

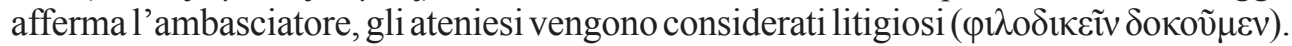
Grote è certamente influenzato da un pregiudizio positivo, poiché ritiene che i processi

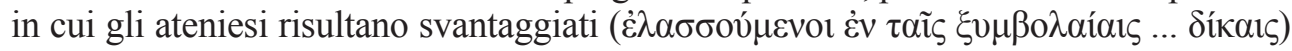

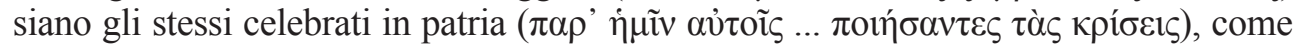
nel caso Fabrigas vs Mostyn. L'immagine che emerge dal passo è però più complessa, poiché, se è vero che nelle dispute regolate da un trattato gli ateniesi non risultavano favoriti, d'altra parte alcune cause furono trasferite d'autorità ad Atene, che pure garantiva un trattamento equo, facendo valere per tutti le stesse leggi.

L'accordo stipulato fra Atene e Faselide alla metà del V secolo a.C., ${ }^{103}$ che Grote non conosceva, ${ }^{104}$ sembra riguardare il primo dei due casi, cioè i processi áxò $\xi v \mu \beta{ }^{\prime} \lambda \omega v$. Secondo de Ste. Croix, il testo (11.6-10) afferma che qualora ad Atene sorgesse una controversia con i faseliti, gli ateniesi concedevano loro di avvalersi di un tribunale presieduto dall' arconte polemarco, il quale trattava solo i casi "involving

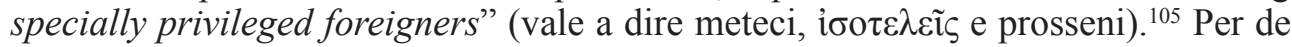
Ste. Croix la controversia poteva svolgersi ad Atene o a Faselide, ma nel caso in cui la sede fosse Atene, i faseliti avrebbero potuto contare su un trattamento di favore. Anche chi non concorda nel dettaglio con questa lettura, ammette però che il decreto è più una concessione che un'imposizione. ${ }^{106}$ Altri decreti attici, ugualmente ignoti a Grote, attestano il trasferimento di alcuni processi ad Atene, ovvero potrebbero riguardare la seconda fattispecie contemplata da Th. 1.77.1. Agli abitanti di Eritre, ad esempio, gli ateniesi ordinano di sorteggiare una boulè di centoventi uomini e prescrivono loro di non accogliere gli esiliati né di bandire i loro concittadini senza il consenso del popolo ateniese; ${ }^{107}$ ai calcidesi invece garantiscono che non potranno deportarli né privarli dei diritti o esiliarli e imprigionarli, senza che siano stati sottoposti a un processo pubblico presso i tribunali ateniesi, ma in compenso $\mathrm{i}$ calcidesi giurano di non ribellarsi al popolo ateniese e di obbedirgli; ${ }^{108}$ allo stesso

100 Grote 1888 (IV), 527-528 n. 1. Di recente, sul significato di symbolaion: Harris 2015, 8-12.

101 Boeckh 1842 (III), 403 n. 417.

102 Meiggs 1972, 232-233.

$103 I G \mathrm{I}^{3}$ 10. Hopper 1943, 39-45; de Ste. Croix 1961, 100-108; Meiggs 1972, 231-232; Fornara 1979; Cataldi 1983, 99-143; Pébarthe 2007.

104 Liddel 2009, 20-27, definisce e circoscrive attentamente l'influenza che questo deficit di conoscenza (unito al pregiudizio negativo nei confronti delle fonti epigrafiche) ebbe nella stesura della History di Grote. Si veda anche Liddel 2014b.

105 de Ste. Croix 1961, 100 (corsivo nell'originale); così già Hopper 1943, 43-44.

106 Meiggs 1972, 232; Fornara 1979, 52; Cataldi 1983, 122-123; Pébarthe 2007, 259.

$107 I G \mathrm{i}^{3}$ 14.8-9, 21-29. de Ste. Croix 1961, 104-105 n. 7; Meiggs 1972, 206; Rhodes 1985, 16; Liddel 2010, 116122; Low 2013, 39-43.

$108 I G \mathrm{i}^{3}$ 40.4-12, 21-32. de Ste. Croix 1961, $271-272$ (in part. su $I G \mathrm{I}^{3}$ 40.71-76); Meiggs 1972, 224-226; Ostwald 2002; Lambert 2017, 19-31. 
giuramento sono vincolate anche Colofone e Samo. ${ }^{109}$ Sulla datazione dei decreti gli studiosi non esprimono una visione unanime. Finora la tendenza generale è stata di collocarli alla metà del V secolo, ma alcuni optano per una datazione più bassa e li pongono nel periodo della guerra archidamica. ${ }^{110} \mathrm{Ci}$ si può chiedere quale sarebbe stata l'interpretazione di Grote, ma probabilmente egli avrebbe scelto l'età di Pericle, poiché la considerava il momento più alto dello sviluppo civile, politico e morale della storia dei greci. Peraltro, le informazioni che possiamo ricavare da questi decreti, che pure arricchiscono il quadro dei rapporti fra Atene e gli alleati, non contraddicono nella sostanza la ricostruzione di Grote: essi attestano che la dominazione ateniese, forse già durante la Pentecontetia, aveva sviluppato forme di controllo abbastanza raffinate, fondate sulla supremazia nell'esercizio della legge, oltre che sulla forza militare.

Certo Grote ha una concezione eccessivamente positiva dell'impero marittimo: in linea con Lewis, egli dà poco peso al tributo come forma di dipendenza ${ }^{111}$ ed è talmente convinto della superiorità degli ateniesi che giunge a postulare un'obbedienza spontanea al dominio di Atene, in cui democrazia e impero finirono per essere due facce della stessa sovranità popolare. ${ }^{12}$ Occasionalmente potevano verificarsi delle rivolte, ma esse non erano da attribuire al demos, che per Grote era per lo più indifferente all'impero, bensì agli oligarchi, che erano avidi di potere. ${ }^{113}$ Grote spiega le rivendicazioni nazionaliste contro il dominio ateniese con la volontà egemonica di alcune minoranze locali, non con l'intolleranza degli ateniesi. Questo sarebbe avvenuto, per esempio, a Samo nel 440-439 a.C. e a Mitilene nel 427 a.C., dove c'erano delle oligarchie. ${ }^{114}$ Durante la guerra del Peloponneso i rapporti fra

$109 I G \mathrm{i}^{3} 37 ; I G \mathrm{i}^{3}$ 48. Sul decreto di Samo si vedano anche l'edizione e il commento di Matthaiou 2014.

110 In particolare per una datazione più bassa di $I G \mathrm{I}^{3} 37$ (Colofone) e 40 (Calcide): Mattingly 1961 (Calcide); 1963 , 266-267 (Colofone); 1992, 132-133 (Colofone), 135-136 (Calcide); 2002 (Calcide); 2014 (Calcide). I primi due interventi sono stati ripubblicati in Mattingly 1996, 53-67, 87-106, un volume in cui lo studioso raccoglie anche altri saggi. Il suo assunto di fondo è che la trasformazione della lega in impero sia avvenuta più tardi, a partire dal decreto di Samo ( $I G \mathrm{I}^{3} 48$ del 439 a.C.). Una delle obiezioni principali alle ipotesi di datazione di Mattingly (e alla sua ricostruzione storica) consisteva nel fatto che i decreti in questione avevano il "three-barred sigma", una forma di scrittura che si riteneva fosse stata abbandonata solo dopo il 446 a.C. (Meiggs 1966); tuttavia l'intervento di Chambers et alii 1990 ha fatto cadere questa convinzione, permettendo di riconsiderare le supposizioni di Mattingly, che oggi non sono più isolate (Moroo 2014; Tracy 2016), per quanto la tendenza sia ancora di accettare datazioni più alte e quindi un assetto imperialista della lega alla metà del V secolo: status quaestionis in Rhodes 1985, 15-17 e 20-21 nn. 19-33; 2008; Kallet 2009, 50-56; Lambert 2017, $28-31$. Sull'espansione ateniese e la creazione dell'impero si veda la sintesi di Samons 2010, 15-28.

111 Grote è contro l'idea che durante la guerra archidamica il tributo abbia raggiunto almeno i 1.200 talenti, come invece sosteneva Boeckh: Grote 1888 (IV), 490-492, 492-493 n. 3; Boeckh 1842 (III), 396-402, 433-440. Le fonti epigrafiche attestano invece che nel 425/424 a.C. il tributo arrivò almeno a 1.460 talenti (IG $\mathrm{I}^{3} 71$; Fornara 1983, n. 136; Meiggs 1972, 324-339; Samons 2000, 173-183; Liddel 2009, 21; Lambert 2017, 32-43), per quanto questa sia più una stima presunta che una somma reale.

112 Grote 1888 (IV), 495: "And altogether the exercise of empire abroad became a prominent feature in Athenian life, and a necessity to Athenian sentiment, not less than democracy at home ... She (sc. Athens) was a capital or imperial city ... with many dependencies attached to her, and bound to follow her orders". Nel definire l'espansione del dominio ateniese (fondazione di colonie e cleruchie) Grote (1888 (IV), 495-510) segue la narrazione di Plutarco e sottolinea la vocazione panellenica e imperiale della politica estera di Pericle: Plu. Per. 11.4-6 (politica coloniale), 12-13 (politica monumentale), 17-28 (politica estera).

113 Grote 1888 (IV), 511-517.

114 Th. 3.27.2-3 racconta che a Mitilene il popolo si ribella ai capi, quando questi gli danno le armi per combattere contro gli ateniesi: ciò potrebbe attestare che in città vigesse un regime oligarchico; quanto a Samo, Th. 8.21 sembrerebbe confermare che nel 412 a.C. essa fosse ancora un'oligarchia, che fu rovesciata solo nel corso di quell'anno (cf. Th. 8.73.2), a meno di non supporre ulteriori cambiamenti avvenuti dopo la fine della rivolta nel 439 a.C. (Th. 1.115-117), ma di cui non siamo a conoscenza: Grote 1888 (IV), 515. Tuttavia l'ipotesi che Samo 
Atene e gli alleati divennero più problematici, poiché le divisioni fra i pochi e $\mathrm{i}$ molti all'interno delle poleis furono esacerbate dal conflitto: i primi tendevano a schierarsi con Sparta, i secondi con Atene. ${ }^{115}$ Per questo nel 412 a.C., durante la riunione dei congiurati a Samo, Frinico avrebbe sconsigliato gli oligarchi ateniesi di sovvertire i regimi democratici nelle città alleate, perché alla fine esse si sarebbero ribellate, come accadde a Taso e in altre località. ${ }^{116}$ Grote crede che fosse più vantaggioso imporre la democrazia che l'oligarchia, perché il demos non era animato dal desiderio di potere, ma pensava al proprio esclusivo tornaconto, opponendosi ai privilegi e ai soprusi dei pochi. ${ }^{117}$ Lungi dall'essere un motivo di lagnanza (come il pagamento del tributo o la presenza delle cleruchie), ${ }^{118}$ l'obbligo di rivolgersi ai tribunali ateniesi costituiva, secondo Grote, un indubbio vantaggio per i sudditi. Infatti Tucidide afferma (per bocca di Frinico) che i popoli sottomessi avevano l'interesse a essere giudicati dai tribunali popolari ateniesi, presso i quali si attendevano una clemenza maggiore che da un regime aristocratico. ${ }^{119}$ Su questo tema si è tornati a discutere grazie a de Ste. Croix, ${ }^{120}$ che ha riaffermato la validità del punto di vista di Grote. In genere gli studiosi esprimono una visione decisamente più cauta, se non addirittura contraria, ma alcuni non escludono che la gestione democratica del potere fosse gradita almeno fra le classi popolari. ${ }^{121}$ La popolarità dell'impero marittimo non è più trattata nei termini entusiastici di Grote, ma la sua lettura ha comunque conservato una certa attualità.

Vi è tuttavia anche un altro aspetto che va considerato ed è il realismo con cui Grote affronta la questione della supremazia giudiziaria di Atene: ${ }^{122}$ questa non stravolgeva

alla fine del V secolo fosse un'oligarchia è stata abbandonata, mentre a Mitilene nel 427 a.C. probabilmente era in vigore un'oligarchia moderata: Gehrke 1985, 117-120, 369-370 (Mitilene), 140-144, in particolare 143 n. 13 (Samo).

115 Th. 3.82.1-2; Grote 1888 (IV), 518.

116 Th. 8.48.5, 64. Grote 1888 (IV), 519. Sulla defezione di Taso: Gehrke 1985, 159-161.

117 Grote 1888 (V), 319-321.

118 Grote 1888 (IV), 520-521.

119 Th. 8.48.6; Grote 1888 (IV), 524 n. 1. Grote si basa anche su [X.] Ath. 1.14-16, in cui il Vecchio Oligarca afferma che l'interesse degli ateniesi è favorire nei tribunali il demos degli stati sottomessi e perseguitare gli aristocratici, che invece sarebbero più propensi a ribellarsi.

120 La tesi della popolarità dell'impero viene esposta in un primo intervento degli anni Cinquanta (de Ste. Croix 2008, in particolare 272-276) e ribadita qualche anno dopo in de Ste. Croix 1972, 34-49, di cui cito un passo: "The evidence is insufficient to enable us to decide how much exploitation and oppression took place in the Athenian empire. No doubt there was a good deal -imperialism is not a pleasant thing. But I suggest that many humble men among the allies had good reason to fear their own oligarchs ... far more than the Athenians, whose deeply rooted belief in democracy and equality before the law made concerned in principle to protect the mass of the people, however much they might on occasion sacrifice their principles to their own greed or desire for power or security" (44). Gli argomenti di de Ste. Croix, in particolare l'uguaglianza di fronte alla legge, influenzano anche Meiggs 1972, 203-233, in particolare 207: "More important than military control by small garrison were the various forms of political control devised by Athens. The most consistent defence of their empire made with conviction by the Athenians was that they relied very little on force. The point is made with special emphasis in the speech of an Athenian envoy to the Spartan Assembly in 432, as reported by Thucydides: the stronger may be expected to use force to control the weaker, but Athens has relied on the processes of law". Per una visione positiva dell'impero anche Pleket 1963 (che però limita la sua indagine a Taso) e più di recente Ober 2015, 217-220.

121 Molto critici sono, ad esempio, Bradeen 1960; Fornara 1977. Assumono invece una posizione intermedia (ammettono cioè la possibilità che l'impero non dispiacesse alle classi popolari, per quanto esprimano forti perplessità sull'intera ricostruzione di de Ste. Croix) Quinn 1964; Rhodes 1985, 36-45; 2007. Per un sunto della questione: Kallet 2009, 44-50.

122. Grote 1888 (IV), 521-522, ritiene che il trasferimento del tesoro della lega da Delo ad Atene inauguri anche l'estensione della giurisdizione ateniese nelle città dell'impero. 
l'assetto costituzionale degli stati sudditi, non aveva cioè il fine di emendare l'amministrazione della giustizia nelle singole città dell'impero, bensì di regolare

... the relations between city and city, between citizens of different cities, between Athenian citizens of officers, and any of these allied cities with which they had relations, between each city itself, as a dependent government with contending political parties, and the imperial Athens. ${ }^{123}$

I tribunali popolari erano un mezzo di governo formidabile per gestire nel modo migliore i rapporti con le dependencies, facendo in modo che esse restassero tali, mantenessero cioè almeno una parvenza di autonomia, senza essere assorbite completamente nella sfera d'influenza ateniese, senza cioè che fosse compromessa la loro natura e l'impero venisse trasformato in qualcosa di diverso da un government of dependencies. A differenza di Sparta, che dislocò le decarchie protette da guarnigioni di armosti, esponendo i sudditi a insopportabili vessazioni, Atene non occupava le città, anche se la sua flotta e i suoi magistrati si spostavano da una parte all'altra dell'Egeo. ${ }^{124}$ Le istituzioni democratiche ateniesi garantivano un regime elastico e tollerabile, poiché i tribunali popolari erano allo stesso tempo una forma di controllo e di garanzia per il popolo, il quale dava voce alle proprie lamentele con la consolazione di essere almeno ascoltato. ${ }^{125}$ Questa sequenza di passi è particolarmente significativa:

If the popular dikasteries at Athens had not been thus open, the allied cities would have suffered much more severely from the captains and officials of Athens in their individual capacity. And the maintenance of political harmony, between the imperial city and the subject ally, was ensured by Athens through the jurisdiction of her dikasteries with much less cost of injustice and violence than by Sparta.

... the Athenian dikasteries, with universal competence and public trial, afforded the best redress which the contingency admitted.

Athens, if she robbed her subject-allies of their independence, at least gave them in exchange the advantage of a central and common judiciary authority. ${ }^{126}$

Come è chiaro soprattutto da quest'ultimo brano, l'impero si fondava su uno scambio di interessi: la perdita dell'indipendenza era compensata da un'autorità centrale e, almeno idealmente, imparziale. Grote legge in chiave filo-ateniese anche le rivendicazioni dei mitilenesi a Olimpia (nel 427), ${ }^{127}$ quando questi si lamentarono con gli spartani delle condizioni a cui erano sottoposti. I loro argomenti sarebbero deboli e confermerebbero che Atene aveva l'egemonia assoluta grazie alla solidarietà delle comunità più piccole, le quali avevano rinunciato alla loro autonomia in cambio del benessere e della sicurezza. Nella visione di Grote Atene è come il Leviatano di Hobbes, al quale i sudditi cedono la loro libertà in cambio della protezione:

123 Grote 1888 (IV), 523.

124 Sui magistrati che venivano inviati a scopo ispettivo nelle poleis alleate: Meiggs 1972, 212-215; Rhodes 1985, 39; Sickinger 2007.

125 Grote 1888 (IV), 523-524.

126 Grote 1888 (IV), 524-525.

127 Th. 3.9-14, in particolare 3.10.5, 3.11.3. 
A city like Mitylene might be strong enough to protect itself and its own commerce without the help of Athens. But to the weaker allies, the breaking up of the Athenian empire would have greatly lessened the security both of individuals and of commerce, in the waters of the Aegean, and their freedom would thus been purchased at the cost of considerable positive disadvantages. ${ }^{128}$

\section{Conclusione}

In conclusione, riassumo i punti salienti di questo intervento. La formazione politica e filosofica di Grote influenza senz'altro la sua interpretazione della democrazia ateniese, che è un sistema di vita prima ancora che un assetto costituzionale. Per Grote la democrazia non implica solo la partecipazione attiva e consapevole del popolo, ma consiste anche nel rispetto della legge e nell'amor di patria. I cittadini cooperano per il bene comune, poiché nel corso degli anni hanno acquisito un alto senso morale grazie alle riforme democratiche. La virtù politica non è innata, ma si può insegnare con un'opportuna educazione, come afferma Platone nel Protagora, uno dei dialoghi preferiti di Grote e dei filosofi radicali inglesi. L'acquisizione di una moralità pubblica rende lo stato più saldo e più stabile, in quanto inibisce le divisioni fra le classi e la frammentazione politica. Grote considera l'oligarchia come la peggiore forma di governo, poiché fa prevalere gli interessi di una minoranza su quelli della maggioranza e indebolisce la comunità acuendo i conflitti interni. Grote riconosce delle analogie fra la monarchia, il governo assoluto di un sovrano, e un'efficace democrazia rappresentativa: entrambe sopiscono gli interessi di parte perché tendono a livellare le differenze.

La recensione del 1839 all'edizione di Hobbes curata da Molesworth dimostra che il progressismo di Grote si nutre anche di realismo politico, il quale influenza la sua ricostruzione della storia ateniese del V secolo. La creazione dell'impero è descritta come un processo graduale, privo di un disegno predeterminato ma con un esito grandioso, al quale cooperano le riforme democratiche di Efialte e Pericle. I nuovi tribunali popolari consolidano la sovranità popolare, che esercita un potere assoluto non solo ad Atene, ma anche nelle comunità dell'impero. Questo è uno degli aspetti più degni di nota dei capitoli 44-47 della History of Greece, nei quali la democrazia radicale viene presentata come il mezzo più efficace per gestire i rapporti con i sudditi. Grote sottolinea la centralità del potere giudiziario, nel quale il demos degli stati sottomessi, che pure vengono privati della libertà, può riscontrare una sorta di garanzia fondata sulla legge, sperando in un trattamento imparziale.

Grote non conosce i decreti attici della metà del V secolo a.C., che trasferivano il giudizio su alcuni reati dai tribunali locali a quelli ateniesi, ed ha quindi una visione meno dettagliata del fenomeno, che è senz'altro più complesso di quanto lasciano intendere le fonti storiografiche. Egli dà una grande importanza alla giurisdizione anche perché guarda all'Inghilterra e viene influenzato dal saggio di George Cornewall Lewis, in cui l'impero marittimo ateniese e l'impero britannico sono entrambi definiti governments of dependencies. Come Lewis, Grote vede profonde somiglianze fra la dominazione ateniese e quella inglese: gli stati sudditi sono sorvegliati dalla flotta e controllati da pubblici ufficiali, ma possono ricorrere ai

128 Grote 1888 (IV), 531. 
tribunali se ritengono di essere trattati ingiustamente. Una parvenza di autonomia nella giurisdizione degli affari interni è garantita, ma nei rapporti internazionali l'ultima parola spetta alla comunità dominante. Grote ritiene che l'impero ateniese fosse più equanime dell'impero britannico: non più invasivo, ma più giusto. Il regime democratico costituisce un valore aggiunto, poiché le giurie popolari sono più clementi. Il processo Fabrigas vs Mostyn, in cui il suddito ha la meglio sul governatore grazie a un tribunale inglese, è una sorta di eccezione nella casistica illustrata da Lewis, ma per Grote esemplifica il livello di equità dei tribunali ateniesi:

The prodigious sum of damages awarded by the jury shows the strength of their sympaty with this Minorquin plaintiff against the English officer. I doubt not that the feeling of the dikastery at Athens was much of the same kind, and often quite as strong; sincerely disposed to protect the subject-allies against misconduct of Athenian trierarchs or inspectors. ${ }^{129}$

I pareri legali sul caso Fabrigas, che sopra abbiamo riassunto, sono paragonati ai dibattiti che si svolgevano ad Atene, dove gli oratori attestano lo scontento degli alleati come una condizione naturale, ${ }^{130}$ alla quale Atene, come Londra, non poteva sperare di sottrarsi. Questi sentimenti sorgono laddove vi è un rapporto fra governi dominanti e governi subordinati, un rapporto necessariamente "uncomfortable"; ma "they are not the product of peculiar cruelty and oppression on the part of Athenian democracy". L'impero marittimo ateniese rispecchia in meglio la realtà dell'impero britannico, ma questo ne rimane pur sempre l'unico riflesso possibile nel presente.

\section{Bibliografia}

Bain, A. (1873): The Minor Works of George Grote, London.

Baker, J. (2003): The Oxford History of the Laws of England. Volume VI: 1483-1558, Oxford (http://dx.doi.org/10.1093/acprof:oso/9780198258179.001.0001).

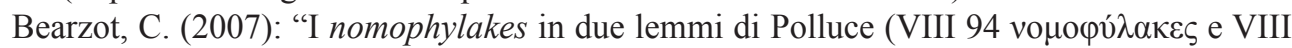
102 oi है $v \delta \varepsilon \kappa \alpha$ ), [en] C. Bearzot et alii (eds.), L'Onomasticon di Giulio Polluce: tra lessicografia e antiquaria (=V\&P. Storia/Ricerche, Contributi di Storia Antica 5), Milano, 43-67.

Bebbington, D. W. (2008): "Gladstone and the Classics”, [en] L. Hardwick - C. Stray (eds.), A Companion to Classical Reception, Malden, 86-97 (http://dx.doi. org/10.1002/9780470696507).

Biagini, E. F. (1992): Il liberalismo popolare. Radicali, movimento operaio e politica nazionale in Gran Bretagna 1860-1880, Bologna.

Boeckh, A. (1842): The Public Economy of Athens, London (1 $\left.{ }^{\mathrm{a}} \mathrm{ed} .1828\right)$.

Boegehold, A. L. et alii (1995): The Lawcourts at Athens. Sites, Buildings, Equipment, Procedure, and Testimonia, Princeton (http://dx.doi.org/10.2307/3602008).

129 Grote 1888 (IV), 533 n. 3.

130 Il riferimento più ovvio è il discorso di Diodoto in occasione del dibattito in assemblea sulla ribellione di Mitilene (Th. 3.42-48, in particolare 45-46), su cui Marcaccini 2014. Sappiamo inoltre che Antifonte pronunciò orazioni in difesa di Lindo e di Samotracia, continuando forse la politica di opposizione di Tucidide di Melesia (Plu. Per. 12.1-2, 14.1): Meiggs 1972, 240-241; Ostwald 1986, 187-188, 363. 
Bradeen, D. W. (1960): “The Popularity of the Athenian Empire”, Historia 9/3, 257-269.

Burroughs, P. (1999): "Imperial Institutions and the Government of Empire," [en] Porter (ed.), 1999, 170-197 (http://dx.doi.org/10.1093/acprof:oso/9780198205654.003.0009).

Calder III, W. M. - Trzaskoma, S. (eds.), (1996): George Grote Reconsidered. A 200th Birthday Celebration with a First Edition of his Essay of the Athenian Government, Hildesheim.

Calder III, W. M. et alii (eds.), (2002): Teaching the English Wissenschaft. The Letters of Sir George Cornewall Lewis to Karl Otfried Müller (1828-1839), (=Spudasmata 85), Hildesheim.

Canevaro, $\mathrm{M}$.

(2018): "Athenian Constitutionalism: Nomothesia and the Graphe Nomon me Epitedeion Theinai”, [en] G. Thür et alii (eds.), Symposion 2017: Vorträge Zur Griechischen Und Hellenistischen Rechtsgeschichte, Wien, 65-98 (http://dx.doi.org/10.2307/j. ctvd7w7mm.9).

(c.s.): Demostene e la libertà greca, da Democare di Leuconoe a Piero Treves, Pisa.

Canfora, L. (2011): Il mondo di Atene, Roma.

Cataldi, S. (1983): Symbolai e relazioni tra le città greche nel V secolo a.C. (=Relazioni interstatali nel mondo antico. Fonti e Studi 4), Pisa.

Chambers, M. (1996): “George Grote's History of Greece”, [en] Calder - Trzaskoma (eds.), 1996, 1-22.

Chambers, M. et alii (1990): “Athens' Alliance with Egesta in the Year of Antiphon", Zeitschrift für Papyrologie und Epigraphik 83, 38-63.

Constantakopoulou, C. (2007): The Dance of the Islands. Insularity, Networks, the Athenian Empire and the Aegean World, Oxford (http://dx.doi.org/10.1093/acprof:o so/9780199215959.001.0001).

Cornish, W. et alii (2010): The Oxford History of the Laws of England. Volume XI: 18201914 English Legal System, Oxford (http://dx.doi.org/10.1093/acprof:o so/9780199258819.001.0001).

Demetriou, K. N. (ed.), (2014): Brill's Companion to George Grote and the Classical Tradition, Leiden (http://dx.doi.org/10.1163/9789004280496).

de Ste. Croix, G. E. M.

(1956): "The Constitution of the Five Thousand", Historia 5, 1-23.

(1961): "Notes on Jurisdiction in the Athenian Empire. I-II", Classical Quarterly 11, 94112, 268-280 (http://dx.doi.org/10.1017/S0009838800015573).

(1972): The Origins of the Peloponnesian War, London.

(2008): “The Character of the Athenian Empire", [en] Low (ed.), 2008, 232-276 (=Historia 3, 1953/4, 1-41).

Ensor, R. C. K. (1936): England 1870-1914. The Oxford History of England, vol. XIV, Oxford.

Figueira, T. (1998): The Power of Money. Coinage and Politics in the Athenian Empire, Philadelphia.

Fornara, C. W.

(1977): "IG I' $\mathrm{I}^{2}, 39.52-57$ and the "Popularity" of the Athenian Empire", California Studies in Classical Antiquity 10, 39-55 (http://dx.doi.org/10.2307/25010712).

(1979): “The Phaselis Decree”, Classical Quarterly 29, 49-52 (http://dx.doi.org/10.1017/ S0009838800035138).

(1983): Archaic Times to the End of the Peloponnesian War, Cambridge (http://dx.doi. org/10.1017/CBO9780511607387). 
Furley, W. D. (1996): Andokides and the Herms. A Study of Crisis in the Fifht-Century Athenian Religion (=Bulletin of the Institute of Classical Studies of the University of London. Suppl. 65), London.

Gagné, R. (2009): "Mystery Inquisitors: Performance, Authority, and Sacrilege at Eleusis", Classical Antiquity 28, 211-247 (http://dx.doi.org/10.1525/ CA.2009.28.2.211).

Gallucci, R. F. (1986): Myth of the Hoplite Oligarchy: Athens, 411/410 B.C., Diss. University of California, Los Angeles.

Gehrke, H. J. (1985): Stasis. Untersuchungen zu den inneren Kriegen in den griechischen Staaten des 5. und 4. Jahrhunderts v.Chr (=Vestigia 35), München.

Gibbins, J. R. (2014): “George Grote and Natural Religion”, [en] Demetriou (ed.), 2014, 85116 (http://dx.doi.org/10.1163/9789004280496_005).

Graf, F. (2000): "Der Mysterienprozess", [en] L. Burckhardt - J. von Ungern-Sternberg (eds.), Grosse Prozesse im antiken Athen, München, 114-127.

Grote, G.

(1847): Seven Letters on the Recent Politics of Switzerland, London.

(1888): A History of Greece, vol. I-X, London.

(1865): Plato, and the Other Companion of Sokrates, vol. I-III, London.

(1873): The Personal Life of George Grote, London.

Hansen, M. H.

(1990): "When was Selection by Lot of Magistrates Introduced in Athens?", Classica et Mediaevalia 41, 55-61.

(1991): The Athenian Democracy in the Age of Demosthenes. Structure, Principles and Ideology, Oxford.

(2001): Graphe paranomon. La sovranità del tribunale popolare ad Atene nel IV secolo a.C. e l'azione pubblica contro proposte incostituzionali, Torino.

Harris, E. M. (2015): "The Meaning of the Legal Term Symbolaion, the Law about Dikai Emporikai and the Role of the Paragraphe Procedure", Dike 18, 7-36 (https://doi. org/10.13130/1128-8221/8025).

Harrison, A. R. W. (1971): The Law of Athens. Procedure, Oxford.

Hopper, R. J. (1943): "Interstate Juridical Agreements in the Athenian Empire", Journal of Hellenic Studies 63, 35-51 (http://dx.doi.org/10.2307/627002).

Hornblower, S.

(1991): A Commentary on Thucydides, vol. I, Oxford.

(2008): A Commentary on Thucydides, vol. III, Oxford.

Huxley, G. (1996): “George Grote on Early Greece”, [en] Calder - Trzaskoma (eds.), 1996, 23-42.

Jenkyns, R. (2007): “United Kingdom”, [en] Kallendorf (ed.), 2007, 265-278 (http://dx.doi. org/10.1002/9780470996775.ch19).

Kahn, C. H. (1996): “George Grote's Plato and the Companion of Sokrates”, [en] Calder Trzaskoma (eds.), 1996, 43-58.

Kallendorf, C. W. (ed.), (2007): A Companion to the Classical Tradition, Malden (http://dx. doi.org/10.1002/9780470996775).

Kallet, L. (2009): "Democracy, Empire and Epigraphy in the Twentieth Century", [en] Ma et alii (eds.), 2009, 43-66.

Kassel, R. - Austin, C. (eds.), (1988): Poetae comici graeci, vol. V, Berlin.

Kenyon, F. G. (ed.), (1891): Aristotle on the Constitution of Athens, Oxford.

Kierstead, J. (2014): “Grote's Athens: The Character of Democracy”, [en] Demetriou (ed.), 2014, 161-210 (http://dx.doi.org/10.1163/9789004280496_008). 
Kinzer, B. (2014): “George Grote, the Philosophic Radical and Politician”, [en] Demetriou (ed.), 2014, 16-46 (http://dx.doi.org/10.1163/9789004280496_003).

Lambert, S. D. (2017): "Two Inscribed Documents of the Athenian Empire. The Chalkis Decree and the Tribute Reassessment Decree", AIO Papers 8, 1-43.

Lewis, G. C.

(1841): An Essay on the Government of Dependencies, London.

(1996): Qual è la miglior forma di governo?, Palermo (1 $\left.{ }^{\mathrm{a}} \mathrm{ed} .1863\right)$.

Liddel, $\mathrm{P}$.

(2006): "Liberty and Obligations in George Grote's Athens", Polis 23, 139-161 (http:// dx.doi.org/10.1163/20512996-90000090).

(2009): "European Colonialist Perspectives on Athenian Power: Before and after the Epigraphic Explosion", [en] Ma et alii (eds.), 2009, 13-42.

(2010): "Epigraphy, Legislation, and Power within the Athenian Empire", Bulletin of the Institute of Classical Studies 53, 99-123 (http://dx.doi. org/10.1111/j.2041-5370.2010.00005.x).

(2014a): "The Comparative Approach in Grote's History of Greece", [en] Demetriou (ed.), 2014, 211-254 (http://dx.doi.org/10.1163/9789004280496_009).

(2014b): "From Chronography to Liberal Imperialism: Greek Inscriptions, the History of Greece, and Historiography from Selden to Grote", Journal of the History of Collections 26, 387-398 (http://dx.doi.org/10.1093/jhc/fhu016).

Loizides, A. (2014): "James Mill and George Grote: A Benthamite Defence of 'Theoretic Reform"”, [en] Demetriou (ed.), 2014, 47-84 (http://dx.doi. org/10.1163/9789004280496_004).

Low, P. (2013): "Law, Authority and Legitimacy in the Athenian Empire", [en] J. Duindam et alii (eds.), Law and Empire. Ideas, Practices, Actors, Leiden, 25-44 (http://dx.doi. org/10.1163/9789004249516_003).

Low, P. (ed.), (2008): The Athenian Empire, Edinburgh.

Ma, J. et alii (eds.), (2009): Interpreting the Athenian Empire, London.

Mann, W. R. - de Harven, V. (2018): "Pleasure, Pain, and the Unity of the Soul in Plato's Protagoras", [en] W. V. Harris (ed.), Pain and Pleasure in Classical Times (=Columbia Studies in the Classical Tradition 44), Leiden, 111-138 (https://doi. org/10.1163/9789004379503).

Marcaccini, C.

(2012): Atene sovietica. Democrazia antica e rivoluzione comunista (=Sentieri 3), Pisa.

(2013): "Rivoluzione oligarchica o restaurazione della democrazia? I Cinquemila, la

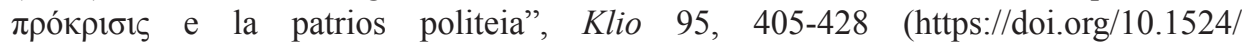
klio.2013.95.2.405).

(2014): "Politica e passioni in Tucidide: il dibattito su Mitilene", [in] A. Campi - S. De Luca (a.c.), Il realismo politico. Figure, concetti, prospettive di ricerca, Soveria Mannelli. (2017): Il conflitto delle élites. Atene 508-403 a.C. (=Mimesis. Eterotopie 410), Milano.

Matthaiou, A. P. (2014): “The Treaty of Athens with Samos ( $\left.I G \mathrm{I}^{3} 48\right)$ ", [en] Matthaiou - Pitt (eds.), 2014, 141-169.

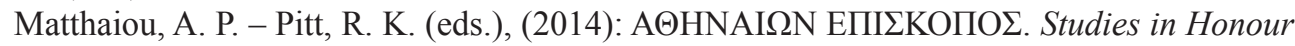
of H.B. Mattingly, Athens.

Mattingly, H. B.

(1961): “Athens and Euboea", Journal of Hellenic Studies 81, 124-132 (http://dx.doi. org/10.2307/628082).

(1963): "The Growth of Athenian Imperialism”, Historia 12/3, 257-273. 
(1992): "Epigraphy and the Athenian Empire", Historia 41/2, 129-138.

(1996): The Athenian Empire Restored. Epigraphic and Historical Studies, Ann Arbor.

(2002): “The Athenian Decree for Chalkis (IG 13 3 .40)", Classical Quarterly 52/1, 377-379 (https://doi.org/10.1093/cq/52.1.377).

(2014): "Athenian Imperialism and the Chalkis Decree (IG I3 40)", [en] Matthaiou - Pitt (eds.), 2014, 11-18.

McGregor, M. (1987): The Athenians and their Empire, Vancouver.

Meiggs, R.

(1966): “The Dating of Fifth-Century Attic Inscriptions", Journal of Hellenic Studies 86, 86-98 (http://dx.doi.org/10.2307/628996).

(1972): The Athenian Empire, Oxford.

Meineke, A. (1839): Fragmenta Comicorum Graecorum, vol. I, Berolini.

Mill, J. S. (1978): “Grote's Plato”, [en] J. M. Robson (ed.), Collected Works of John Stuart Mill, vol. XI: Essays on Philosophy and the Classics, Toronto, 377-440.

Momigliano, A. (1955): "George Grote and the Study of Greek History", [en] A. Momigliano (ed.), Contributo alla storia degli studi classici (=Storia e Letteratura 47), Roma, 215-231.

Moroo, A. (2014): “The Erythrai Decrees Reconsidered: $I G \mathrm{I}^{3}$ 14, 15 \& 16", [en] Matthaiou - Pitt (eds.), 2014, 97-119.

Murray, O. (2004): “A Lost School of History: Ancient Greece in the Age of Reform”, [en] O. Murray (ed.), Edward Bulwer Lytton, Athens: Its Rise and Fall. With Views of the Literature, Philosophy, and Social Life of the Athenian People, Bicentenary Edition, London-New York, 1-34.

Ober, J.

(1996): The Athenian Revolution. Essays on Ancient Greek Democracy and Political Theory, Princeton.

(2007): “'I Besieged That Man'. Democracy’s Revolutionary Start”, [en] Raaflaub et alii (eds.), 2007, 83-104 (http://dx.doi.org/10.1525/california/9780520245624.003.0004).

(2015): The Rise and Fall of Classical Greece (=The Princeton History of the Ancient World 1), Princeton (http://dx.doi.org/10.1515/9781400865550).

Ostwald, M.

(1986): From Popular Sovereignty to the Sovereignty of Law. Law, Society, and Politics in Fifth-Century Athens, Berkeley-Los Angeles-London.

(2002): "Athens and Chalkis: A Study in Imperial Control", Journal of Hellenic Studies 122, 134-143 (http://dx.doi.org/10.2307/3246208).

Pappè, H. O. (1979): “The English Utilitarians and Athenian Democracy", [en] R. R. Bolgar (ed.), Classical Influences on Western Thought: A.D. 1650-1870, Cambridge, 295-307.

Pébarthe, C. (2007): “Contrats et justice dans l'empire athénien: les symbolaia dans le décret d'Athènes relatif à Phasélis $\left(I G, \mathrm{I}^{3}, 10\right)$ ", [en] P. Brun (ed.), Scripta anatolica. Hommages à Pierre Debord (=Ausonius Éditions. Études 18), Paris, 237-260 (http://dx.doi. org/10.4000/books.ausonius.11330).

Pleket, H. W. (1963): "Thasos and the Popularity of the Athenian Empire”, Historia 12/1, 70-77.

Porter, A. (1999): “Trusteeship, Anti-Slavery, and Humanitarianism”, [en] Porter (ed.), 1999, 198-221 (http://dx.doi.org/10.1093/acprof:oso/9780198205654.003.0010).

Porter, A. (ed.), (1999): The Oxford History of the British Empire, Volume III: The Nineteenth Century, Oxford (http://dx.doi.org/10.1093/acprof:oso/9780198205654.001.0001).

Preyer, R. O. (1982): “John Stuart Mill on the Utility of Classical Greece”, Browning Institute Studies 10, 41-70 (http://dx.doi.org/10.1017/S0092472500006829). 
Quinn, T. J. (1964): “Thucydides and the Unpopularity of the Athenian Empire", Historia $13 / 3,257-266$.

Raaflaub, K. A. (2007): “The Breakthrough of Demokratia in Mid-Fifth-Century Athens", [en] Raaflaub et alii (eds.), 2007, 105-154 (http://dx.doi.org/10.1525/california/ 9780520245624.003.0005).

Raaflaub, K. A. et alii (eds.), (2007): Origins of Democracy in Ancient Greece, Berkeley-Los Angeles-London (http://dx.doi.org/10.1525/california/9780520245624.001.0001).

Richardson, S. (2014): “A Regular Politician in Breeches: The Life and Work of Harriet Lewin Grote", [en] Demetriou (ed.), 2014, 134-160 (http://dx.doi.org/10.1163/ 9789004280496_007).

Rhodes, P. J.

(1972): "The Five Thousand in the Athenian Revolution of 411 B.C.", Journal of Hellenic Studies 92, 115-127 (http://dx.doi.org/10.2307/629977).

(1985): The Athenian Empire (=Greece \& Rome. New surveys in the classics 17), Oxford. (1992): A Commentary on the Aristotelian Athenaion politeia, Oxford.

(2007): "Democracy and Empire", [en] Samons (ed.), 2007, 24-45 (http://dx.doi. org/10.1017/CCOL9780521807937.002).

(2008): "After the Three-Bar Sigma Controversy: The History of Athenian Imperialism Reassessed", Classical Quarterly 58/2, 500-506 (https://doi.org/10.1017/ S000983880800058X).

Samons II, L. J.

(2000): Empire of the Owl. Athenian Imperial Finance (=Historia. Einzelschriften 142), Stuttgart.

(2010): “Athens: A Democratic Empire", [en] K. Kagan (ed.), The Imperial Moment, Cambridge, 12-31.

Samons II, L. J. (ed.), (2007): The Cambridge Companion to the Age of Pericles, Cambridge (https://doi.org/10.1017/CCOL9780521807937).

Sancho Rocher, L.

(2015): "La Historia de Grecia de Georges Grote y la Atenas de los liberales", [en] L. Sancho Rocher (coord.), La antigüedad como paradigma. Espejismos, mitos y silencios en el uso de la historia del mundo clásico por los modernos, Zaragoza, 87-119.

(2018): “¿Es la demokratía semejante a la democracia? Lecturas contemporáneas de la democracia ateniense", Logos 51, 15-33 (http://dx.doi.org/10.5209/ASEM.61641).

Sartori, F. (1957): Le eterie nella vita politica ateniese del VI e V secolo a.C., Roma.

Schömann, G. F.

(1854): Die Verfassungsgeschichte Athens nach G. Grote's History of Greece kritisch geprüft, Leipzig.

(1855): Griechische Alterthümer, I. Das Staatswesen, Berlin.

(1878): Athenian Constitutional History, as represented in Grote's History of Greece critically examined, Oxford.

(1897): Griechische Alterthümer, I. Das Staatswesen, Berlin.

Sealey, R. (1975): "Constitutional Changes in Athens in 410 B.C.", California Studies in Classical Antiquity 8, 271-295 (http://dx.doi.org/10.2307/25010694).

Sickinger, J. P. (2007): "The Bureaucracy of Democracy and Empire”, [en] Samons (ed.), 2007, 196-214 (http://dx.doi.org/10.1017/CCOL9780521807937.009).

Strauss, B. S. (1996): "The Athenian Trireme, School of Democracy", [en] J. Ober - C. Hedrick (eds.), Demokratia. A Conversation on Democracies, Ancient and Modern, Princeton, 313-325. 
Tracy, S. V. (2016): Athenian Lettering of the Fifth Century B.C. The Rise of the Professional Letter Cutter, Berlin (https://doi.org/10.1515/9783110407594).

Traill, J. S.

(1975): The Political Organisation of Attica. A Study of the Demes, Trittyes and Phylai, and their Representation in the Athenian Council (=Hesperia Supplement 14), Princeton (https://doi.org/10.2307/1353928).

(1986): Demos and Trittys. Epigraphical and Topographical Studies in the Organization of Attica, Toronto.

Tritle, L. A.

(1999): “The Athens of George Grote: Historiography and Philosophic Radicalism”, [en] R. Mellor-L. Tritle (eds.), Text and Tradition: Studies in Greek History and Historiography in Honor of Mortimer Chambers, Claremont, 167-178.

(2013): "Democracy and War", [en] J. P. Arnason et alii (eds.), The Greek Polis and the Invention of Democracy, Chichester, 298-320 (http://dx.doi.org/10.1002/9781118561768. ch13).

Turner, F. M. (1981): The Greek Heritage in Victorian Britain, New Haven-London.

Urbinati, N. (2006): L'ethos della democrazia. Mill e la libertà degli antichi e dei moderni (=Biblioteca di cultura moderna 1186), Roma.

Vance, N. (2007): "Victorian", [en] Kallendorf (ed.), 2007, 87-100 (http://dx.doi. org/10.1002/9780470996775.ch8).

van Wees, H. (2007): "War and Society", [en] P. Sabin - H. van Wees - M. Whitby (eds.), Cambridge History of Greek and Roman Warfare. Volume 1. Greece, The Hellenistic World and the Rise of Rome, Cambridge, 273-299 (http://dx.doi.org/10.1017/ CHOL9780521782739.010).

Whedbee, K. E. (2004): "Reclaiming Rhetorical Democracy: George Grote's Defense of Cleon and the Athenian Demagogues", Rhetoric Society Quarterly 34/4, 71-95 (http:// dx.doi.org/10.1080/02773940409391296).

Whitehead, D. (1986): The Demes of Attica 508/7 - ca. 250 B.C. A Political and Social Study, Princeton (http://dx.doi.org/10.1515/9781400857685).

Woodward, E. L. (1938): The Age of Reform 1815-1870. The Oxford History of England, vol. XIII, Oxford.

Zuckert, C. (2014): “Grote's Plato”, [en] Demetriou (ed.), 2014, 273-302 (http://dx.doi. org/10.1163/9789004280496_011). 